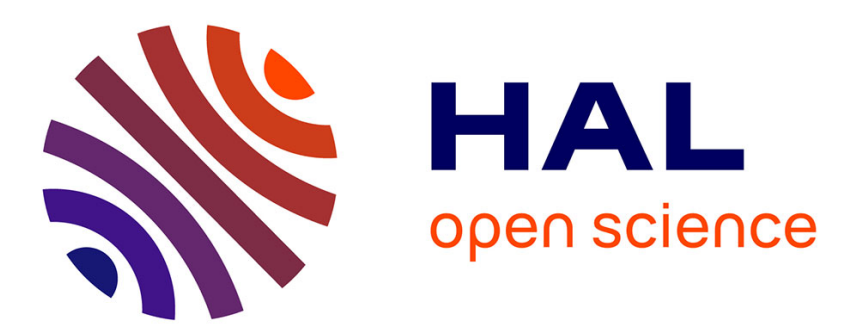

\title{
Optical Index Prism Sensitivity of Surface Plasmon Resonance Imaging in Gas Phase: Experiment versus Theory
}

Jonathan Shilanthan Weerakkody, Sophie Brenet, Thierry Livache, Cyril Herrier, Yanxia Hou, Arnaud Buhot

\section{To cite this version:}

Jonathan Shilanthan Weerakkody, Sophie Brenet, Thierry Livache, Cyril Herrier, Yanxia Hou, et al.. Optical Index Prism Sensitivity of Surface Plasmon Resonance Imaging in Gas Phase: Experiment versus Theory. Journal of Physical Chemistry C, 2020, 124 (6), pp.3756-3767. 10.1021/acs.jpcc.9b09973 . hal-02485279

\section{HAL Id: hal-02485279 \\ https://hal.science/hal-02485279}

Submitted on 23 Sep 2020

HAL is a multi-disciplinary open access archive for the deposit and dissemination of scientific research documents, whether they are published or not. The documents may come from teaching and research institutions in France or abroad, or from public or private research centers.
L'archive ouverte pluridisciplinaire HAL, est destinée au dépôt et à la diffusion de documents scientifiques de niveau recherche, publiés ou non, émanant des établissements d'enseignement et de recherche français ou étrangers, des laboratoires publics ou privés. 


\title{
Optical Index Prism Sensitivity of Surface Plasmon Resonance Imaging in Gas Phase: Experiment vs. Theory
}

\author{
Jonathan S. Weerakkody, ${ }^{\dagger}$ Sophie Brenet, ${ }^{\dagger}$ Thierry Livache, ${ }^{\ddagger}$ Cyril Herrier,,$^{\ddagger}$ \\ Yanxia Hou, ${ }^{\dagger}$ and Arnaud Buhot ${ }^{*, \dagger}$ \\ $\dagger$ Univ. Grenoble Alpes, CEA, CNRS, IRIG, SyMMES, 17 Rue des Martyrs, 38000 \\ Grenoble, France \\ $\ddagger$ Aryballe Technologies, F-38000 Grenoble, France \\ E-mail: arnaud.buhot@cea.fr
}

\begin{abstract}
Recent advances in SPR-imaging detection in gas phase have led to the development of opto-electronic noses (opto-eNs) requiring the need for optical sensitivity characterization of such devices. Understanding of the optical contributions will have an implicit relevance on sensitivity enhancement of SPR-imaging in gas phase valuable to improve the performance of opto-eN and potentially open new applications as gas sensors. In this paper, we analyzed the optical contributions to the sensitivity of the SPR imaging prisms and potential insights into their contributing factors. We established a characterization method for the SPR prism sensitivity that is independent of the carrier gas considered. Then, by using this sensitivity parameter, the influence of two different kinds of adhesive layers, $\mathrm{Cr}$ and $\mathrm{Ti}$, of the gold coated prisms were studied. Furthermore, we considered a theoretical model to rationalize our experimental results, which demonstrated the relevance of surface topography on the optical index
\end{abstract}


sensitivity. Those surface topographies were characterized experimentally and were implemented in the model free from any additional fitting parameters using a modified Maxwell-Garnet theory. Finally, the model was shown to predictively assess the experimentally measured effects of temperature on the prism sensitivity.

\section{Introduction}

Surface Plasmon Resonance (SPR) is an optical technique widely used in biosensor development for the sensing of chemical and biological compounds. $\frac{12}{2}$ The effectiveness of this technique can be attributed to its high sensitivity, real-time monitoring, label-free, and high throughput analysis. SPR based sensing techniques have been studied extensively over the past two decade for biomolecular interaction analysis in liquid phase. These SPR biosensors are based on various interrogation techniques, namely: angular, wavelength, phase, polarization and intensity. ${ }^{3}$ Intensity interrogation based SPR allows for an interrogation of the full surface of the prism by a camera also known as SPR imaging and is particularly interesting due to its multi-parametric detection capabilities. In this study, we will be focusing on this interrogation technique for gas phase analysis where the intensity of the reflected light will be referred to as its reflectivity.

As mentioned, SPR imaging systems are mostly used in liquid based affinity sensing. ${ }^{2}$ However, other interrogation techniques such as angular interrogation have been adapted for gas phase sensing in several studies over the years. ${ }^{[4]}$ A recent article published by Brenet et al. highlights, for the first time, the efficiency of SPR imaging for the detection and discrimination of Volatile Organic Compounds (VOCs) in gas phase. ${ }^{7}$ Such a SPR imaging system has demonstrated great promise for a novel opto-electronic nose (opto-eN)! ${ }^{17-10}$ Among its many advantages over existing eN systems, SPR imaging detection provides multiplexing capabilities allowing for the simultaneous monitoring of a large sensor array in real time. Furthermore, enhanced discriminatory capabilities can be seen through the temporal response of reflectivity changes, allowing for the extraction of multiple defining parameters, 
such as equilibrium and adsorption and desorption kinetic responses for the generation of robust VOC fingerprint patterns. $\frac{7111}{1 n}$ addition to high selectivity, eNs are also associated with high sensitivities showing low limit of detection (LOD), for certain VOCs, comparable to a human nose. ${ }^{9}$ Sensitivity enhancement remains a pertinent interest as it would not only improve the detection limits for trace levels of VOCs but also aid in discriminatory purposes by increasing contrast of the aforementioned fingerprints. In response to this necessity, it is important to first characterize such SPR imaging systems. This process involves the contribution of two major aspects, namely, the optical and chemical contributions to the system sensitivity. The chemical contribution is attributed to the biomolecular interactions between the VOCs and the obtained sensing surfaces; either with semi-conductor surfaces, $\frac{122}{,}$ polymer layers ${ }^{13}$ or surface functionalized with biomolecular receptors. ${ }^{\frac{114115}{}}$ In comparison, the optical contribution involves the SPR phenomenon itself and depends on the device parameters such as wavelength, temperature and metal film thicknesses. Before focusing on improving the chemical performances by selecting the optimal receptors for VOCs detection, it remains crucial to first optimize the experimental set-up and particularly the SPR prism coatings in terms of optical performances.

In this study, we will be focusing on the optical contribution to the systems' sensitivity. Thus, before the preparation of the sensor array on the SPR imaging prisms, they need to be optically characterized. A robust technique referred to in this paper as the sensitivity parameter approach was established as a performance measurement for the SPR imaging set-up. This measure was shown to be independent of the carrier gas used allowing for prism calibration and further compensation for environmental variations during in-situ or on-field measurements. Furthermore, using this performance parameter, the prism materials and fabrication contributions to these experimentally derived values were evaluated and compared with a theoretical model. Two different adhesive layers, Chromium $(\mathrm{Cr})$ and Titanium (Ti), were studied for their impact on the overall performance. The subsequent deposition of the gold $(\mathrm{Au})$ layer on the glass prism led to further surface topography considerations, which 
were analyzed using Atomic Force Microscopy (AFM). Even though there have been previous studies focused on surface plasmon enhancement, by reducing surface roughness, via processes such as annealing $\frac{16}{16}$ It remains a challenge, specifically, trying to understand the contributions of these surface topographies. We demonstrated that the surface topography (the $z$-direction surface roughness and $x$-, $y$-direction lateral contributions) of the Au layer played a major role in the sensitivity losses due to damping effects. This was tested using the AFM measurements of the surface topography, where a new approach was validated for the incorporation of these surface topography damping effects into the theoretical model. A well known mixing formulae was used for an effective layer accounting for the roughness of the Au layer which was experimentally validated free from any fitting parameters. Finally, the impact of 3-dimensional surface topographies on the optical index sensitivity were confirmed with a better understanding of their possible implications. In consequence, the theoretical model was predictive for the temperature variation of the performances; thus, being useful in proposing recommendations for sensitivity improvements and drift corrections for the opto-eN. By decoupling this optical temperature contribution it would be possible to further understand the complexities of this compounded temperature effect on the subsequently immobilized receptors.

\section{Theoretical Considerations}

For the implementation of the theoretical model a multi-layer Kretchmann configuration was employed mimicking the experimental system being used. ${ }^{17}$ In agreement with this approach, our SPR imaging prism was considered to consist of $N$-layers. Therefore, to obtain the reflectivity in this multi-layer system, the transfer matrix method was considered. This formalism was first established in 1950 by Abeles, $\frac{18}{18}$ which was further expanded in 1968 by Hansen. $\frac{19}{19}$ The transfer matrix approach highlighted by Hansen was closely followed in this study (see Supporting Information). 
The significance of this approach lies in its simplicity to accurately and precisely determine the reflectivity with the absence of approximations. Each layer of the model is considered to be stacked on the $z$-direction defined as a function of its complex relative permittivity $\epsilon_{N}$ (square of its optical index $n$ ), its complex relative permeability $\mu_{N}$ and its thickness $d_{N}$. The summarized idea of this multi-layer matrix method is schematically represented in figure 11. Nevertheless, the drawback of this method arises from the assumption that these layers are considered to be homogeneous, isotropic and plane-bounded. Thus, the roughness of the successive layers is not taken into account.

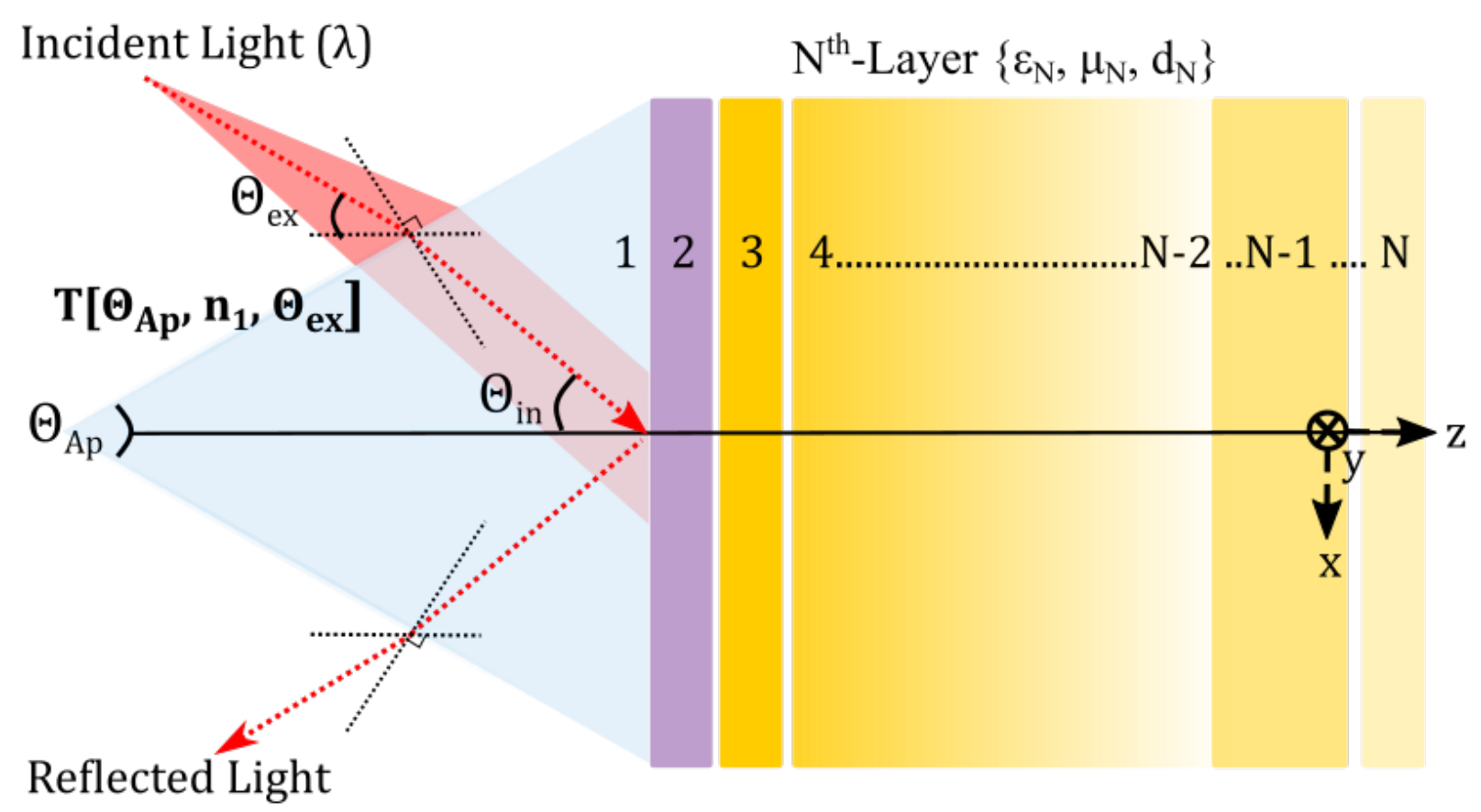

Figure 1: Schematic representation of the multi-layer system used for the calculation of the reflectivity. Incident light, with a specific wavelength $(\lambda)$, is considered with transmission losses through the prism (layer 1) quantified by the transmission function $T$. ${ }^{20}$ For its definition the external incident angle $\left(\theta_{e x}\right)$, prism optical index $\left(n_{1}\right)$ and the Apex angle of the prism $\left(\theta_{A p}\right)$ are used. For the transfer matrix method, the adhesive layer (layer 2) and $\mathrm{Au}$ layer (layer 3) are followed with successive layers defined by their complex relative permittivities $\epsilon_{N}$ (square of their optical index $n$ ), complex relative permeabilities $\mu_{N}$ and their thicknesses $d_{N}$ which is utilized with the internal incident angle $\left(\theta_{i n}\right)$ experimentally equivalent to the working angle $\left(\theta_{w}\right)$. These parameters and there relationships are further explained in the supplementary section.

In accordance, we initially considered a 4 -layer system $(N=4)$. Since only non-magnetic 
materials are present in the model, $\mu_{N}$ is equal to 1 for all the relevant layers. Furthermore, the glass prism made of NBK-7 (layer 1) and the Dry Air carrier gas region (layer 4) are considered to be semi-infinite along the $z$-axis on opposite directions. The Au layer (layer 3 ) is generally attached to the prism through a thin metallic layer of $\mathrm{Cr}$ or $\mathrm{Ti}$ (layer 2) for better adhesion purposes. For all the other layers, except for the thin adhesive layer (due to its low thickness), the effect of temperature was considered when driving its complex relative permittivity $\epsilon_{N}$. To ensure the robustness of the models employed, fitted models were chosen from experimental observations at ambient conditions ${ }^{21}$ and varied temperature studies. $\frac{22}{24}$ In addition, the effect of pressure was introduced into the permittivity function of the gas phase (layer 4) for the theoretical analysis of the system's performance. Table 1 summarizes the 4 layers incorporated into the basic matrix model for the reflectivity. The models used for the permittivity functions of each layer are listed, referencing the experimental studies from which they are derived. The optimum $d_{N}$ values, for increased sensitivity, were chosen based on previous studies showing an adhesive layer thickness of $\approx 2 \mathrm{~nm}$ and an Au layer thickness of $\approx 53 \mathrm{~nm} . \stackrel{25126}{.}$ However, the real obtainable thicknesses, with slight over shot from set values, were experimentally obtained using a Quartz Crystal Microbalance (QCM) in each case.

Table 1: Summary of the information on the layers in the simulated multi-layer system.

\begin{tabular}{llll}
\hline $\mathbf{N}$ & Material & $d_{N}[\mathrm{~nm}]$ & Model \\
\hline \hline 1 & $\mathrm{NBK}-7$ & $\infty$ & $\begin{array}{l}\text { Temperature-dependent Sellmeier equation based } \\
\text { model }{ }^{22}\end{array}$ \\
\hline 2 & $\mathrm{Cr} / \mathrm{Ti}$ & $d_{2}=[0-6]^{*}$ & Brendel-Bormann model 21 \\
\hline 3 & $\mathrm{Au}$ & $d_{3}=54.5^{*}$ & Temperature-dependent Drude-Sommerfeld model [23 \\
\hline 4 & Dry air & $\infty$ & $\begin{array}{l}\text { Temperature \& Pressure-dependent Sellmeier } \\
\text { equation based model }\end{array}$ \\
\hline
\end{tabular}

* Obtained experimentally from QCM measurements during the physical vapor deposition procedure for a set of optimized thickness $\left(d_{2}=2 \mathrm{~nm}, d_{3}=53 \mathrm{~nm}\right)$. 


\section{Surface topography layer}

An important limitation for the matrix model involves the incorporation of the surface topography damping effects. There are only a few studies which have dealt with simulating the effects of surface topography on the SPR phenomenon. ${ }^{27}$ In each of the cases, different approaches were explored. In this work we will be focusing on the approach known as the modified Maxwell-Garnet Theory (mMGT). Taking into account the use of a multi-layer system, the main advantage of mMGT is its specific applicability within the transfer matrix method. In fact, mMGT treats the surface topography as an additional layer within the model. The dielectric function of this effective layer $\left(\epsilon_{e f f}\right)$ was defined in mMGT by relating the dielectric function of the host media $\left(\epsilon_{4}\right)$ with the dielectric function $\left(\epsilon_{3}\right)$, volume fraction $(f)$ and depolarization factor $\left(v_{p}\right)$ of the metal inclusions. Equation 1 relates the effective permittivity of the roughness layer with the modified Maxwell-Garnett mixing formula from the permittivities of the pure phases and accounting for the geometry of the metal inclusions. $\frac{30}{30}$

$$
\epsilon_{e f f}=\epsilon_{4} \Delta \frac{\epsilon_{4}+\left[v_{p}(1-f)+f\right]\left(\epsilon_{3}-\epsilon_{4}\right)}{\epsilon_{4}+v_{p}(1-f)\left(\epsilon_{3}-\epsilon_{4}\right)} \quad p=x, y, z
$$

Due to large variations in metal inclusions when considering topography, a conjecture was made that the mixing formula (Eq. 1) was a valid approximation, as long as, on average, the medium was spatially uniform and anisotropic. $\frac{30}{30}$ The depolarization factor $\left(0<v_{p}<1\right)$ acts as a measure representing the weakening of the internal field due to polarization damping, while also providing an indication of the geometry of the forenamed metal inclusions. The individual depolarization factor coefficients along the three directions follow the normalization condition $v_{x}+v_{y}+v_{z}=1$. To further understand this approximation, we need to assume similarly oriented ellipsoids with semi-axes $a_{x}, a_{y}, a_{z}$ parallel to the Cartesian axes (see figure 1 and figure 5). In the case of prolate ellipsoids, $a_{x}=a_{y} \ll a_{z}$ (geometrically equivalent to thin needle shapes) depolarization factors correspond to $v_{x} \simeq v_{y} \rightarrow 1 / 2$ and $v_{z} \rightarrow 0$. Whereas, for 
oblate ellipsoids, $a_{x}=a_{y} \gg a_{z}$ (geometrically equivalent to flat pancake shapes) $v_{z} \rightarrow 1$ and $v_{x} \simeq v_{y} \rightarrow 0$. General expressions for these geometrically dependent depolarization factors are given by $v_{x}=Q\left(a_{x}, a_{y}, a_{z}\right), v_{y}=Q\left(a_{y}, a_{z}, a_{x}\right)$ and $v_{z}=Q\left(a_{z}, a_{x}, a_{y}\right)$. For the purpose of this study, $v_{z}$ is the only depolarization factor considered, in accordance with the transfer matrix method, as it has most significant contribution to the prism sensitivity . In addition, the most pertinent effect derived from the experimental data is the polarization damping in the $z$-direction of the prism. The following equations were derived for oblate ellipsoids, following the condition of $a_{x}=a_{y}>a_{z}$. 31

$$
Q\left(a_{z}, a_{x}, a_{y}\right)=\frac{a_{x} a_{y}}{2 a_{z}^{2}} \int_{0}^{\infty} H\left(s, a_{z}, a_{x}, a_{y}\right)^{-1 / 2} d s
$$

with

$$
H\left(s, a_{z}, a_{x}, a_{y}\right)=(s+1)^{3}\left(s+\left(\frac{a_{x}}{a_{z}}\right)^{2}\right)\left(s+\left(\frac{a_{y}}{a_{z}}\right)^{2}\right)
$$

\section{Experimental considerations}

\section{Gas phase SPR imaging setup}

The custom-built gas phase SPR imaging system under consideration follows the intensity interrogation approach. Here, the refractive index variations of the gas phase are detected on the basis of a change in the reflectivity at a fixed wavelength and incident angle. ${ }^{32}$ Following this approach, initially, the most suitable operating angle was identified manually with angular scans. Once identified, this working angle $\left(\theta_{w}\right)$ was fixed and the reflectivity $R(t)$ of the region of interest $(\mathrm{ROI})$ was monitored as a function of time. $\theta_{w}$ was usually selected to give the highest slope of the linear region of the plasmon curve, thereby selected close to the angle maximizing $\partial R / \partial \theta$. However, the system only allows for incremental angular shifts of $0.25^{\circ}$, therefore, the closest possible angle within this range was selected. This angular interrogation was performed for every sample being analyzed to ensure maximum achievable 
sensitivity of the system, enabling better characterization of small refractive index changes.

This custom-built setup, schematically represented in figure 2, was equipped with a $625 \mathrm{~nm}$ intensity modulated Light Emitting Diode (LED) (M625F2, Thorlabs). However, since $632.8 \mathrm{~nm}$ wavelength was used for VOC analysis, ${ }^{17}$ a bandpass filter (central wavelength 632.8 $\mathrm{nm}$, bandwidth $3 \mathrm{~nm}$ - FL05632.8-3, Thorlabs) was employed right after the prism to capture the relevant wavelength of reflected light. In relation to the intensity losses induced during transmission through the filter, the power modulator unit (KPS101, Thorlabs) ensured due compensation for these losses. Initially, the beam from the optical fiber was evenly distributed using a diffuser as a means of preventing hot-spots. Then the beam was collimated, to ensure the required ROI was entirely illuminated. Following which the beam was passed through a linear polarizer (extinction ratio > 1000: 1 - LPVISE100-A, Thorlabs) in order to achieve the Transverse Magnetic/Electric (TM/TE) modes required for SPR sensing. Once the beam was reflected off the metal coated prism surface, it is passed through the filter before being finally captured by a 16-bit CCD camera (AVT PIKE F-145B, Allied Vision Technologies), with a resolution of $1388 \times 1038$ pixels. The captured image of the ROI is then constantly monitored at the TM mode. This entire optical setup was placed under a temperature controlled environment (Memmert Oven). The reflectivity of the system was calculated and monitored using a data acquisition and analysis program designed using LabVIEW (National Instruments).

In addition to this optical setup, a fluidic system was employed for controlled gas phase analyses. It was crucial in this study that the pressure and flow were accurately controlled throughout the system. Therefore, a simple fluidic setup was implemented with a massflow controller and a pressure controller (Bronkhorst, Netherlands). The flow was set to $100 \mathrm{ml} / \mathrm{min}$ and a baseline pressure was set 50 mbar above ambient conditions (absolute pressure of 1063.25 mbar ) to ensure carrier gas flow through the head space. Four different carrier gases were analyzed in this study, namely, $\mathrm{Ar}$ (purity 99.99\%), $\mathrm{CO}_{2}$ (purity 99.99\%), He (purity 99.99\%) and Dry Air (<1 ppm of $\mathrm{CO}_{2}+\mathrm{CO},<0.2 \mathrm{ppm}$ of tertiary hydrocarbons 


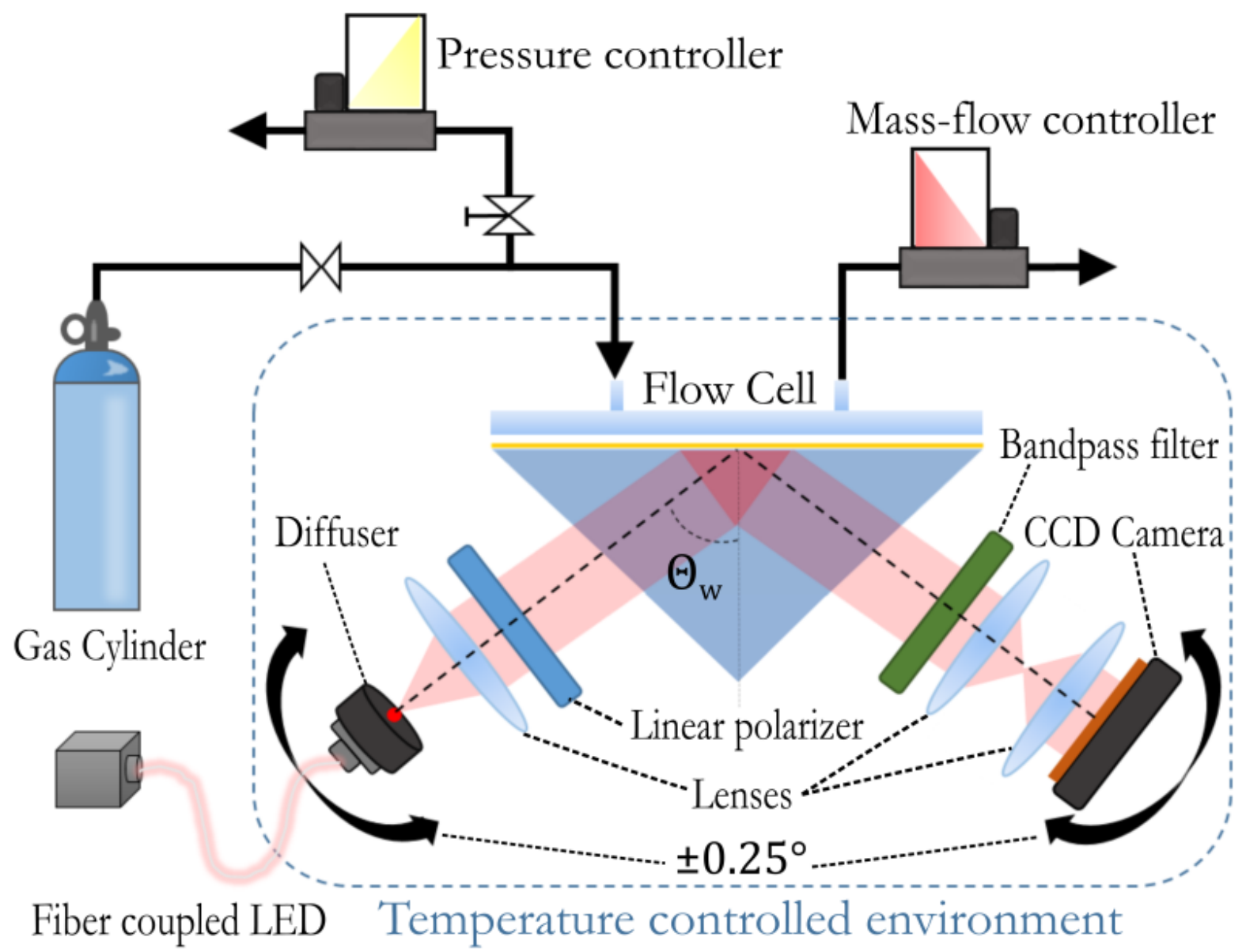

Figure 2: Schematic representation of the custom-built experimental setup. A $625 \mathrm{~nm}$ LED is coupled to an optical fiber, followed by a diffuser, at the point source, through which the light is scattered. The initial lens collimates the beam which is then $s$ - or $p$-polarized (TE and TM modes) via the linear polarizer. The working angle $\left(\theta_{w}\right)$ representing the incident beam perpendicular to metalized surface is calibrated, however, limited by $0.25^{\circ}$ increments. The Flow cell is used to create a pressure lock over the illuminated ROI. The subsequent bandpass filter narrows the spectral distribution to obtain the required working wavelength of $632.8 \mathrm{~nm}$. Followed by the focusing lenses before the image is captured using a 16-bit CCD camera. In addition, the fluidic setup placed outside the temperature controlled environment consists of a pressure and mass flow controller with gas flow and system pressure modulation capabilities. 
and $<3 \mathrm{ppm}$ of $\mathrm{H}_{2} \mathrm{O}$ ). The pressure of these carrier gases were modulated accurately using a control loop feedback mechanism, where the evacuated gas was conserved using a hand operated gate valve.

\section{Metal film deposition on prisms}

Metal film deposition on the active prism surface was performed using an electron (e-) beam evaporator, Plassys MEB 550-S, in a clean-room environment. This physical vapor deposition (PVD) technique was selected for a plethora of advantages pertaining to optical thin film applications. $\frac{33}{3}$ However, in relevance to our study, this method was best suited due to its ambient operating temperatures. In accordance, the sample prisms were kept at low temperatures during Au-film deposition, preventing formation of isolated Au islands prevalent with samples exposed to high temperatures. ${ }^{34}$ In addition, multi-crucible e-beam sources provided the capability to sequentially evaporate metals, preserving the vacuum. This was another unique advantage, as it prevented contamination during the design of multi-layer systems similar to our own. In addition to these immediate benefits, it was also important to consider the future miniaturization and mass production of such gas phase SPR imaging sensor prisms. Thus, e-beam evaporators provided faster deposition rates enabling high efficiencies and low cost production lines. $\frac{35}{35}$

For the preparation of high RI sample prisms, initially, $25 \mathrm{~mm}$ right apex angled $\left(\theta_{A p}=\right.$ 90 $)$, NBK-7 prisms (Edmund optics) were etched with an $\mathrm{Ar}^{+}$ion gun at $250 \mathrm{~V}$ for $24 \mathrm{~s}$ (optimized parameters). This process ensured a clean and considerably flatter surface prior metal deposition (see figure S3). After this etching process the required metal thin film layers were deposited, inline with our setup corresponding to the Kretschmann configuration. The accuracy of the deposition rate and film thickness were determined by QCM, where the reproducibility of the layer thickness was $\pm 1 s$ of the deposition rate.

For this work, three different samples were prepared to analyze the effect of adhesive layers $(\mathrm{Cr}$ or $\mathrm{Ti}$ ) on the surface topography of the thin film Au-layer, with the subsequent 
translation of these topography effects onto the sensitivity of these SPR imaging sensor prisms. A summary of the prepared samples are shown in table 2.

Table 2: Summary of sample prisms prepared using Plassys MEB 550, e-beam evaporator.

\begin{tabular}{llll}
\hline Sample & Deposition Layers & Deposition rates $[\mathrm{nm} / \mathrm{s}]$ & Layer Thickness $[\mathrm{nm}]$ \\
\hline \hline 1 & $\mathrm{Cr} / \mathrm{Au}$ & $0.1 / 0.25$ & $2.4 / 54.5$ \\
\hline 2 & $\mathrm{Ti} / \mathrm{Au}$ & $0.1 / 0.25$ & $3.6 / 54.5$ \\
\hline 3 & $\mathrm{Au}(\mathrm{no}$ adhesive layer) & 0.25 & 54.5 \\
\hline \hline
\end{tabular}

\section{AFM analyses}

For Au surface characterization of the samples shown in table 2, AFM scans were performed using the Dimension Fastscan ${ }^{\mathrm{TM}}$ (Bruker). However, for the visualization of the initial and intermediate stages of the SPR imaging prism preparation, a more commonly used Dimension Icon $^{\mathrm{TM}}$ (Bruker) was used. With the Fastscan ${ }^{\mathrm{TM}}$, surface topography data were acquired in tapping mode under ambient conditions. A higher resolution $\mathrm{Si}_{3} \mathrm{~N}_{4}$ cantilever having an integral tip with spring constant of $17 \mathrm{~N} / \mathrm{m}$ and tip diameter of $5 \mathrm{~nm}$ was used (FASTSCANA, Bruker). Images were obtained by oscillating the cantilever slightly below its resonance frequency $(1400 \mathrm{kHz})$ and raster scanning across the surface. With the Icon $^{\mathrm{TM}}$, a lower resolution $\mathrm{Si}_{3} \mathrm{~N}_{4}$ cantilever having an integral tip with spring constant $0.4 \mathrm{~N} / \mathrm{m}$ and tip diameter of $5 \mathrm{~nm}$ was used (SCANASYST-AIR, Bruker). Images in this case were obtained by ScanAsyst ${ }^{\circledR}$ mode a type of peak force tapping mode ${ }^{\circledR}$.

A $1 \times 1 \mu m^{2}$ area was scanned for each sample at the center of the ROI. The images obtained for each of the samples had a resolution of $512 \times 512$, which were quantified using Gwyddion software. The images were leveled and filtered. Further analyses were performed using the Gwyddion built-in algorithms and then the data were fitted and graphically represented (with an interpolation function of order 3) using the Mathematica computational platform. Note that all the AFM images were plotted at scale $-1 \times 1 \times 0.4$, to better highlight the surface topography. The same scaling was maintained for comparative analyses 
with different color gradients to emphasize the material being represented (see figure [S3).

\section{Results and discussion}

\section{Prism sensitivity characterization}

In order to analyze and compare performances of different SPR imaging prisms, it was imperative to establish a standardized method to characterize the prism samples independent

of the measurement procedure. A sensitivity measure, which is prevalent in literature, 25136 is defined as the reflectivity variation per refractive index unit (RIU) or $\partial R / \partial n$. The utilization of a gas phase SPR imaging system gives us the unique advantage of using pressure as a physical parameter affecting the refractive index of the gas. This dependence was capitalized upon to obtain $\partial R / \partial P$, experimentally from pressure jumps, with various gases. The derivation of the SPR imaging system performance, $\partial R / \partial n$, is then deduced from the calculated values of $\partial P / \partial n$ for the various gases considered following this simple relation:

$$
\frac{\partial R}{\partial n}=\frac{\partial P}{\partial n} \times \frac{\partial R}{\partial P}
$$

To experimentally validate the proposed pressure based sensitivity factor approach, 4 different carrier gases were tested ( $\mathrm{Ar}, \mathrm{CO}_{2}$, He and Dry Air) with the current $\mathrm{Cr} / \mathrm{Au}$ (prism sample 1) configuration used in our opto-eN system. Three pure gases were chosen with the Dry Air mixture to emphasis the robustness of this approach. The experimental approach involved monitoring the change in reflectivity over time at a fixed working angle (close to the one maximizing $\partial R / \partial \theta$ ) and at a constant working temperature of $25^{\circ} \mathrm{C}$. Then, to obtain $\partial R / \partial P$ the pressure was increased, sequentially, from 50 mbar (above ambient pressure) up to 450 mbar at 100 mbar increments. These step-wise pressure jumps were repeated, immediately, in the opposite direction (as shown by the temporal response of the system for Dry Air in the inset of figure 3) to account for any baseline drift in the SPR imaging 
signal. The pressure was maintained at each increment for two minutes following which the signal plateaus, at each pressure point, were averaged to obtain the change in reflectivity with respect to pressure (as shown in figure 3). A strong linear relationship was observed, through which the experimental $\partial R / \partial P$ values were extracted. Five spots (replicates) with diameters of $200 \mu \mathrm{m}$ were chosen and analyzed to accurately represent the entire ROI of the prisms. In addition, the experiments were also repeated twice to ensure the reproducibility and accuracy of the experimentally derived data. In effect, the standard deviation values for $\partial R / \partial n$ accounts for both the replicates and experimental repeats.

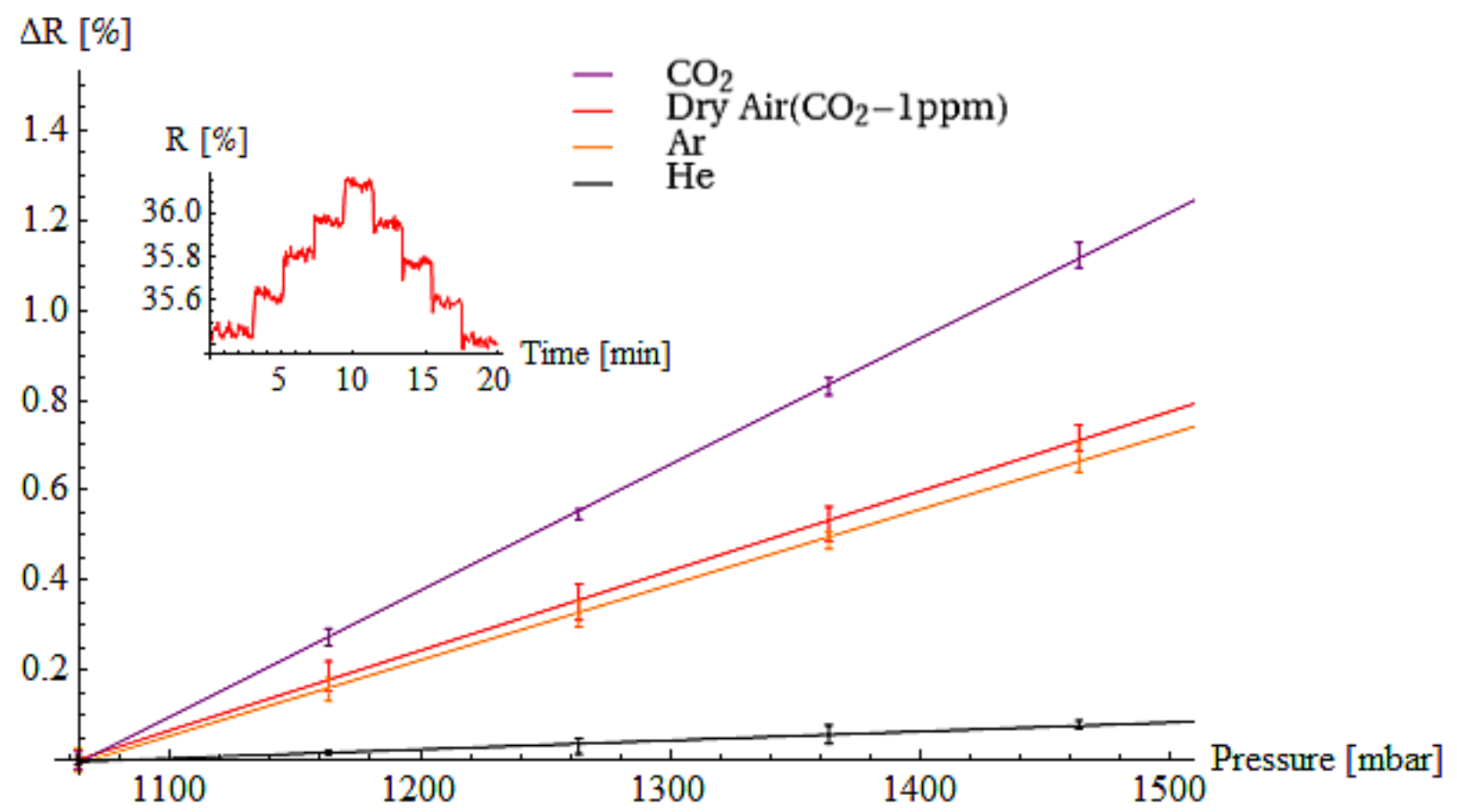

Figure 3: Experimental change in reflectivity $(\Delta R)$ with respect to pressure for the 4 different gases $\left(\mathrm{CO}_{2}\right.$, Dry Air, Ar and He). A linear fit was obtained. Inset - The temporal response of reflectivity for a single repeat pressure scan, showing the 100 mbar pressure increments with Dry Air (visualization of raw data).

As seen in table 3, pure gases/gas mixtures with larger molecular weights had a larger $\partial R / \partial P$. Therefore, in order to determine an independent measurement of the SPR imaging set-up performance, it was important to obtain $\partial R / \partial n$ which is independent of the carrier 
gas being used. The next step along this line was to obtain $\partial n / \partial P$ values. The effect of pressure on the refractive index of pure gases have been well documented. The Hauf-Grigull relation 37 (equation 4) was used to calculate these values for the 3 simple carrier gases, Ar, $\mathrm{CO}_{2}$ and $\mathrm{He}$ :

$$
n(\rho)-1=\frac{3}{2} \frac{r M P}{R T}
$$

where $r$ is the gas refractivity, $M$ is the gas molar mass, $P$ is the pressure, $R$ is the ideal gas constant, and $T$ is the temperature which was set to ambient $\left(25^{\circ} \mathrm{C}\right)$ in our case $\stackrel{38}{\text { For Dry }}$ Air, however, the effect of pressure on its refractive index was a more complex relationship. Since Dry Air was a mixture of gases, it was important to introduce additional parameters, such as humidity and $\mathrm{CO}_{2}$ concentration, to improve the accuracy of the $\partial n / \partial P$ values that needed to be extracted. A robust experimentally verified model proposed by Ciddor ${ }^{24}$ was used for this purpose, where pressure, humidity, $\mathrm{CO}_{2}$ concentration, temperature (and its effects on the saturation vapor pressure) were related to the refractive index change of Air. In the Supplementary Information we present the refractive index variations of the 4 different carrier gases with respect to changes in pressure (figure $\mathrm{S} 1$ ). $\frac{\sqrt[38]{3}}{\mathrm{~T}}$ The parameter $\partial n / \partial P$ were extracted via a linear fit.

Interestingly, we can observe in table 3 that the experimental values for $\partial R / \partial P$ are highly sensitive to the carrier gases utilized. However, when corrected using the pressure dependence on the refractive index $(\partial P / \partial n)$ the overlap of the sensitivity parameter $\partial R / \partial n$ is excellent and lies within error bars for all 4 carrier gases. This validates the use of $\partial R / \partial n$ as a sensitivity parameter for the set-ups performance and allows us to compare performances between various prism samples independent of the carrier gas being used.

The sensitivity of $\mathrm{Cr} / \mathrm{Au}$ prism (sample 1) was considered to be $\partial R / \partial n=6650 \pm$ $60 \% / R I U$, for the default carrier gas, Dry Air. Based on such a performance, we can further analyze the LOD of the SPR imaging system. For this purpose, the noise fluctuations of the reflectivity signal were identified to be approximately $0.05 \%$ (see figure S2). From the minimal signal detectable, corresponding to the level of noise, the minimum resolvable bulk 
Table 3: Values obtained for the sensitivity parameter using prism sample 1 with $\mathrm{Cr} / \mathrm{Au}$ layers. All experiments were carried out at ambient temperature $\left(25^{\circ} \mathrm{C}\right)$.

\begin{tabular}{|c|c|c|c|}
\hline Carrier Gas & $\partial R / \partial P[\% / m b a r]$ & $\partial n / \partial P \quad[R I U / m b a r]$ & $\partial R / \partial n[\% / R I U]$ \\
\hline Carbon dioxide $\left(\mathrm{CO}_{2}\right)$ & $4.21 \times 10^{-7}$ & 0.00279 & $6640 \pm 30$ \\
\hline Dry Air & $2.55 \times 10^{-7}$ & $0.00169^{*}$ & $6650 \pm 60$ \\
\hline Argon (Ar) & $2.51 \times 10^{-7}$ & 0.00166 & $6620 \pm 30$ \\
\hline Helium (He) & $3.13 \times 10^{-8}$ & 0.00021 & $6640 \pm 80$ \\
\hline
\end{tabular}

refractive index change for the $\mathrm{Cr} / \mathrm{Au}$ prism is about $7.5 \times 10^{-6} \mathrm{RIU}$, which is comparable to the LOD of many reported affinity based SPR imaging biosensors in liquid . 1 The relevance of this bulk carrier gas LOD is tied with real world VOC measurements as the carrier gas (Air) composition changes situationally, especially during on-field detection. For instance, a 10\% increase of relative humidity in air leads to an approximate change of $10^{-5} R I U .24$ Thus, $1 \%$ change in humidity could ideally be detected by the SPR imaging set-up and potentially be compensated for. Such correction of external parameters like humidity or temperature would improve the VOC signature leading to more robust and reproducible measurements in varied environmental conditions. Thus, this sensitivity factor approach would be the ideal means to calibrate such SPR imaging prisms before their utilization. And furthermore could be also and important characterization tool for the immobilized receptors on the sensing array.

Similarly, $\partial R / \partial n$ was experimentally extracted for the $\mathrm{Ti} / \mathrm{Au}$ prism sample 2, giving a value of $6490 \pm 120 \% / R I U$ at a similar maximized working angle. The standard deviation for sample 2 was twice that of sample 1, which was a result of the significant variability in experimental replicates. The minimum distinguishable reflectivity (noise) which was about $0.07 \%$ was slightly higher than that of sample 1, leading to a minimal resolvable bulk refractive index change of $10^{-5} R I U$. Moreover, the pressure jump experiments were also performed on prism sample 3 with only a Au layer. However, with no adhesive layer (either Ti or Cr), there was a large signal drift and noise observed (shown in figure S2). As a result, it was not possible to obtain reliable $\partial R / \partial n$ values. 
It was clear from this initial experimental study that adhesive layers played a vital role in obtaining reliable and reproducible results. Furthermore, $\mathrm{Cr} / \mathrm{Au}$ prism sample 1 was identified as the better sensor prism at $25^{\circ} \mathrm{C}$ and working angle $\theta_{w}=42.5^{\circ}$, as it showed higher sensitivities with better reproducibility of performances due to smaller standard deviation values. The comparison between $\mathrm{Ti}$ or $\mathrm{Cr}$ adhesive layers on the performance of the SPR imaging set-up is analyzed in more detail and compared to model predictions in the following sections.

\section{Theoretical comparison of $\mathrm{Cr}$ and $\mathrm{Ti}$ adhesive layers on prism sen- sitivity}

As previously discussed, it is well known that PVD of Au directly onto a glass substrate suffers from weak adherence of the layer due to the low reactivity of similar noble metals. To enhance this adherence usually an adhesive layer $(1-6 \mathrm{~nm})$ of $\mathrm{Cr}$, $\mathrm{Ti}$ or their oxide forms are used.$\underline{39}$ Over the years, significant strides have been made to understand the effects of adhesive layers on the SPR phenomenon. Recent studies have shown that Ti was preferred over $\mathrm{Cr}$ as it absorbed light less strongly and was prone to less variations in transmission 40 (which can be seen in figure 4 - Inset). In addition, comparative studies have shown a localized surface plasmon damping in plasmonic nanostructures due to the presence of a very thin adhesive layer. Overall better performances were noticed for $\mathrm{Ti}$ over $\mathrm{Cr}$ for the nano-optic applications.$\underline{4142}$

To understand this further, the initial 4-layer model was used. In retrospect, it is important to understand that when performing PVD metal depositions, generally, there is an overshoot from the set film thickness. This is usually attributed to the vaporized metal remaining in the deposition chamber. As a consequence, at similar deposition rates, Ti generated a thicker layer compared to $\mathrm{Cr}$ for an optimized set thickness value of $2 \mathrm{~nm}$ (shown in table 22. The vapor pressure of $\mathrm{Ti}$ was higher than that of $\mathrm{Cr}, \underline{43}$ making it more volatile, resulting in a larger amount of metal vapor remaining in the deposition chamber. The de- 
pendence of this adhesive layer thickness $d_{2}$ on the sensitivity parameter $\partial R / \partial n$ is shown in figure 4. Where, it can be inferred that by decreasing $d_{2}$ the sensitivity could be further improved. The rational behind this result is linked to the dielectric permittivities of the metals. For surface plasmon propagation, at certain resonance conditions, the real and imaginary part of the permittivities govern the significance of the plasmonic effect. The real part directly corresponds to the resonance condition whereas the imaginary part corresponds to the internal damping of the transmitted power. Cr and Ti have a considerably larger imaginary part which broadens the spectral width of the plasmon curve at all given wavelengths. Therefore, essentially, to maximise sensitivity a pure Au layer (with the largest plasmonic effect) with no adhesive layer would be ideal. This is validated in figure 4, where a maximum value of $\approx 9500 \% / R I U$ was theoretically predicted for both samples in the simulated model with no adhesive layer. However, as seen earlier with prism sample 3, direct Au deposition onto a NBK-7 prism provided experimentally unreliable results, due to large signal drift and noise. This was expected, as the fluctuations in the signal were related to the gradual detachment of $\mathrm{Au}$ from the NBK-7 prism. Evidence of this weak adherence was physically noticeable in sample 3, which showed heavy signs of delamination and peeling, progressively worsening with an additional time-dependent performance deterioration. In essence, a thickness of $d_{2} \simeq 2 \mathrm{~nm}$ for the anchoring layer (either $\mathrm{Cr}$ or $\mathrm{Ti}$ ) was a good compromise between the complete NBK-7 surface coverage and optimized sensor prism performance.

$\partial R / \partial n$ for $\mathrm{Cr}$ and $\mathrm{Ti}$ based prisms were theoretically determined to be $8170 \% / R I U$ and $8350 \% / R I U$, respectively, after adhesive layers thickness were set (see Figure 4). This was in agreement with previous studies, as it showed a performance enhancement with the use of $\mathrm{Ti}$ as an adhesive layer. However, experimental performances $\partial R / \partial n$ showed much lower sensitivity values at a similarly optimized working angle $\theta_{w} \simeq \theta_{\max }$ where $\theta_{\max }$ corresponds to the angle maximizing the performance $\partial R / \partial n$. These sensitivity losses, attributed to surface topography damping effects, are experimentally validated in the following section. 


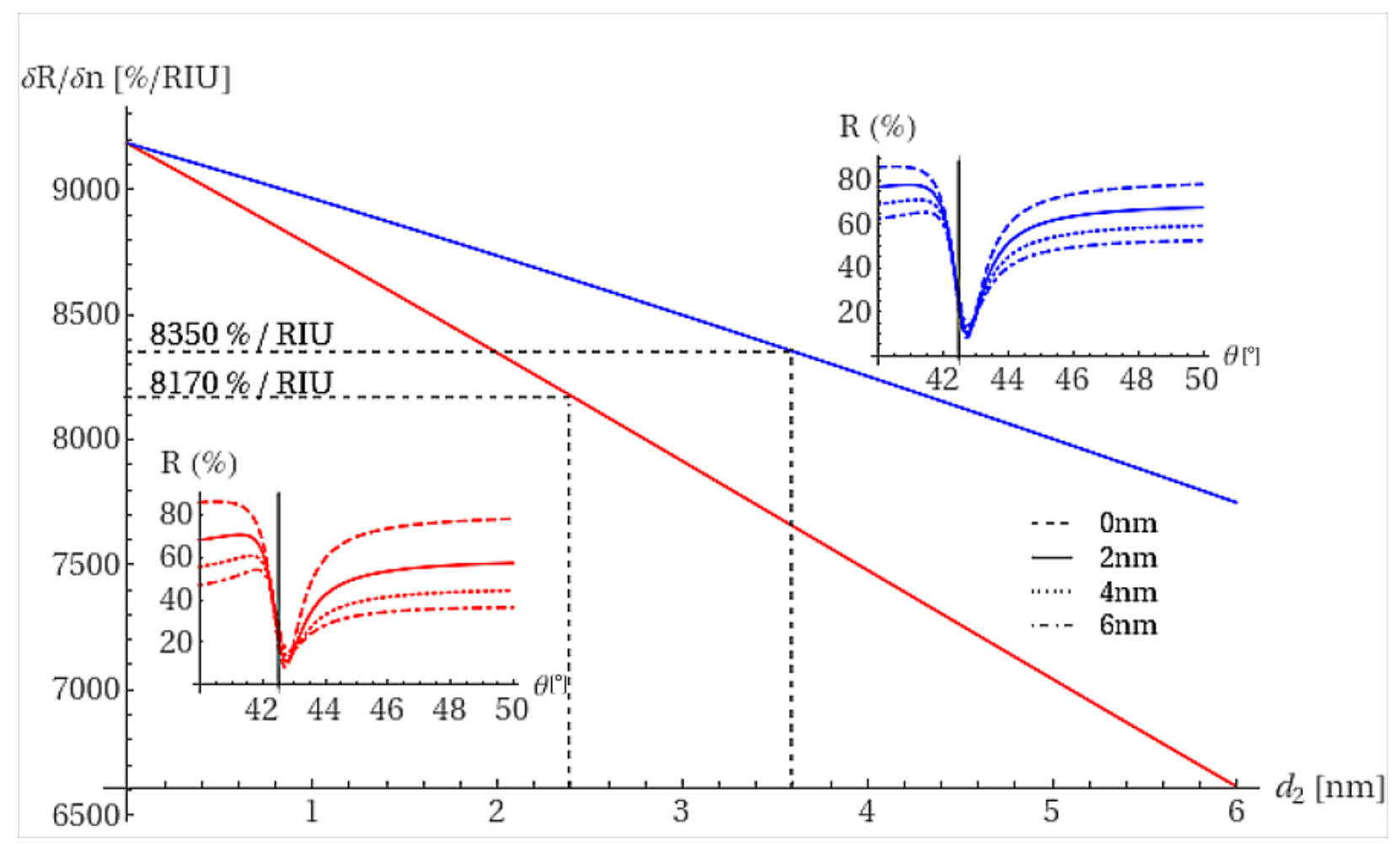

Figure 4: Theoretical $\partial R / \partial n$ for both $\mathrm{Cr}$ (Red) and Ti (Blue) with respect to the thickness of each adhesive layer. Results obtained via the initial 4-layer model; the model was established with $\mathrm{Au}$ thickness generated from the deposition $(54.5 \mathrm{~nm})$ and at an optimized set working angle of $42.5^{\circ}$ for maximized sensitivity. Inset(s) - Reflectivity (\%) with respect to the angle $(\theta)$ for both $\mathrm{Cr}$ (bottom) and $\mathrm{Ti}$ (top) at adhesive layer thicknesses 0,2 , 4, and $6 \mathrm{~nm}$. 


\section{Impact of Surface topography on prism sensitivity}

As seen earlier, when experimentally verified, the sensitivities for $\mathrm{Cr}$ and $\mathrm{Ti}$ adhesive layer prisms were found to be $6650 \% / R I U$ and $6490 \% / R I U$, respectively. These values are significantly lower than the predicted sensitivities for the initial model with the 4-layer system. This system assumes a homogeneous, flat surface. Therefore, it fails to account for any surface topography damping effects that might affect the performance of the system. It is important to characterize these losses as they accounted for an approximate $20 \%$ of reduction in the systems estimated performance. Which, when fully understood, could be further used to enhance our opto-eN system to achieve lower LOD. In order to consider these losses an additional $5^{\text {th }}$ layer, known as the effective layer, was introduced into our theoretical model. This $5^{\text {th }}$ layer was governed by the mMGT. As seen earlier, the mMGT is a mixture model which characterizes the surface topography as a collection of ellipsoidal inclusions on the surface of layer 3 (scheme in figure 5). To correctly implement the dielectric function of this effective layer, it is necessary to first derive two parameters $v_{z}$ and $f$, experimentally. For this purpose, $1 \times 1 \mu \mathrm{m}$ AFM measurements were taken at the center of the ROI for each of the samples (shown in figure 6). In validation of our choices and constraints of the mixing model, randomly distributed regular grains (semi-ellipsoidal in shape) were noticed on the surface of these adhesive layer based samples (1 and 2). This was coherent with another $\mathrm{Ti} / \mathrm{Cr}$ based adhesive layer study, as they were shown to enhance surface wetting leading to

more nucleated grain growth with a narrow grain size distributions ${ }^{422}$ However, for sample 3 , the scans showed no such regularity in grain size or shape which further validated this idea, correlating to its weak adherence. To ensure that the presence or absence of these grains were purely a result of the adhesive layers, AFM measurements were taken along all the intermediate sample preparation steps (before and after etching, and after adhesive layer deposition) (shown in figure S3). It is clear that these semi-ellipsoidal grains can only be seen on the Au surface as a result of the presence of these adhesive layers. The etching seems to play an important role in flattening the surfaces of these NBK-7 prisms, 
as predicted, since the Root-Mean-Squared roughness $\left(R_{q}\right)$ was decreased from $1.06 \mathrm{~nm}$ to $0.60 \mathrm{~nm}$ after etching. Following which, the flatness is shown to be maintained with the adhesive thin film deposition. With even a slight improvement, represented with a small decrease in the $R_{q}(0.50 \mathrm{~nm}$ for $\mathrm{Cr}$ and $0.45 \mathrm{~nm}$ for Ti adhesive layers). However, after the deposition of $\mathrm{Au}$, the $R_{q}$ was seen to increase, drastically, to $1.8 \mathrm{~nm}$ and $1.5 \mathrm{~nm}$ for sample 1 and 2, respectively. In accordance, this increase in roughness was primarily visualized as these aforementioned regular, semi- ellipsoidal grains. For sample $3, R_{q}$ was calculated to be $1.3 \mathrm{~nm}$ which, visually, didn’t have a well defined shape or regularity.

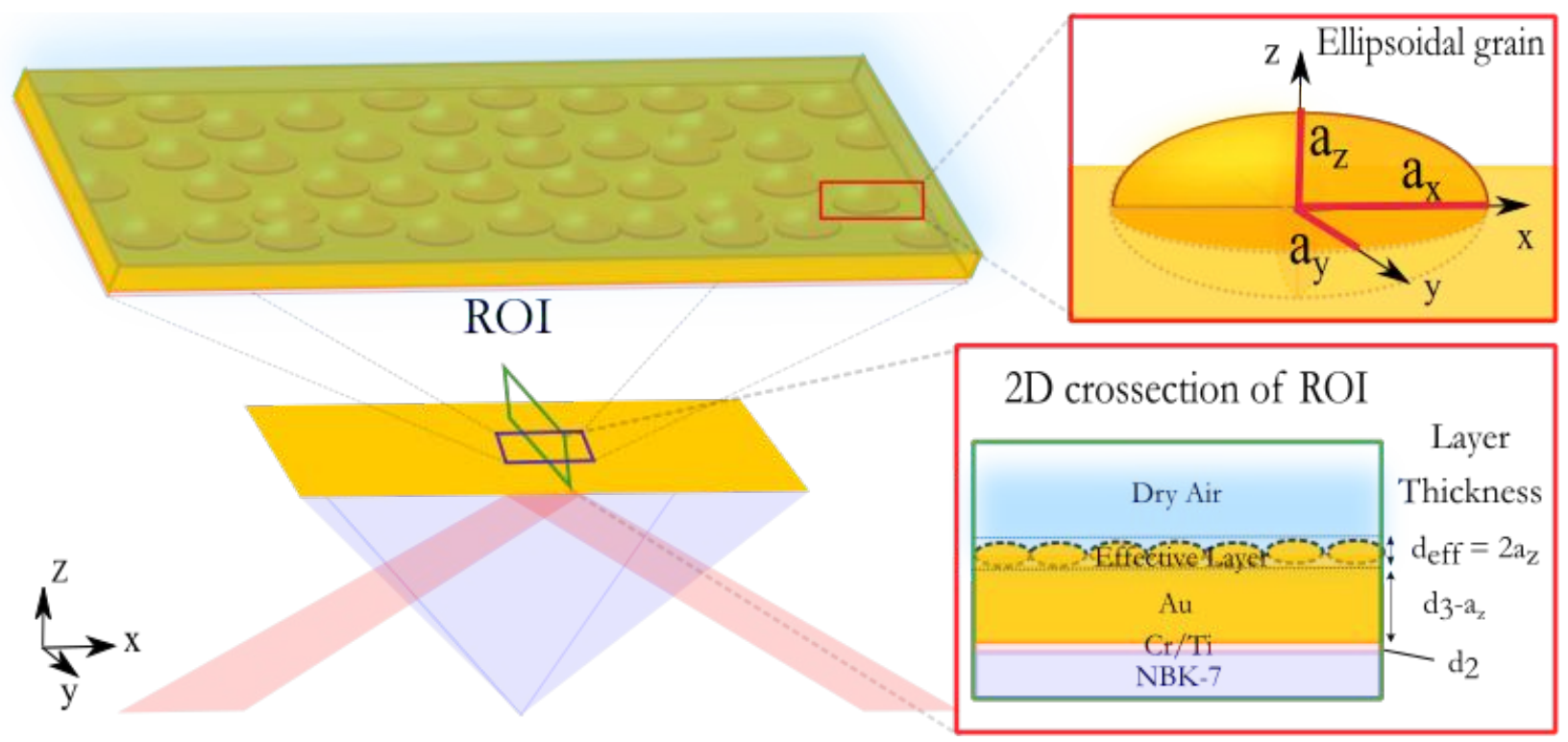

Figure 5: Schematic representation of the application of the mMGT - Au grains are represented as ellipsoidal inclusions (with semi-axes $a_{x}, a_{y}$ and $a_{z}$ parallel to the Cartesian axes) in a Dry Air host media forming our effective layer. The modified layer thicknesses are shown in the $2 \mathrm{D}$ cross-section of the ROI.

Now, before implementing this new 5 -layer system a few considerations need to be made to the initial model. When using the mMGT, grains are visualized to be similarly oriented ellipsoidal inclusions with semi-axes $a_{x}, a_{y}$ and $a_{z}$ parallel to the Cartesian axes. Knowing this, $d_{3}$ needs to be modified by subtracting $a_{z}$ as the QCM measurements only account for the mean value of the deposited height of the Au layer. Following which, the thickness of the effective layer $d_{e f f}$ is also represented with $2 a_{z}$ gauging its complete shape (shown in 
figure 5). Moreover, since the surface topography was considered as an additional layer in the model, the resultant resonance angular shift were compensated for. Furthermore, the additional layer also elongated the plasmon curves corresponding to the internal damping predicted by the model, thus the experimental intensities were also adjusted accordingly. This stays inline with the idea that both these layers are in fact just a single layer exhibiting different plasmonic behaviors reflecting realistic experimental conditions. Following these considerations, for the implementation of the mMGT, 4 new parameters were needed. These included the indicative average dimensions of these ellipsoidal inclusion $\left(a_{x}, a_{y}\right.$ and $\left.a_{z}\right)$ and the estimated volume fraction $f$ of these said inclusions.

Firstly, $a_{z}$ was considered. $a_{z}$ plays a significant part in the derivation of $v_{z}$ and $d_{e f f}$. To find its best representative value, a distribution was constructed from the $z$-axis heights obtained via the AFM surface profiles. The distribution was seen to fit best with a Gaussian function $G$ (equation 5), showing that it was isotropic, corresponding to a certain level of $z$-direction regularity in grain structure. $G$ was fitted with $A$ the amplitude and $\sigma$ the standard deviation of the distribution. $a_{z}$ was determined as $3 \sigma$ which accounted for $\approx 99.7 \%$ of the distribution (see figure S4). Which meant that most of the noise observed in the tails of the Gaussian $(z>3 \sigma)$ and related to measurement technique were ignored, giving a more representative value. This is especially important with $\mathrm{Cr}$ adhesive layer where the trailing ends of the Log-Height distribution were ignored (figure S4 Inset). These trailing ends were related to higher levels of impurities and surface defects seen on this particular sample.

$$
G(z)=A \Delta e^{-\frac{z^{2}}{2 \sigma^{2}}}
$$

$a_{z}$ was calculated for $\mathrm{Cr}$ and Ti samples yielding values of $5.4 \mathrm{~nm}$ and $4.7 \mathrm{~nm}$, respectively. $\sigma$, in our case, also closely related to the $R_{q}$ of the system since our mean height was set to zero ${ }^{44}$ This approach is significant, similar to previous works, $\stackrel{28}{28}$ as it also provides a roughness characterization parameter.

Secondly, in contrast to these previous roughness studies, two other geometric contribu- 

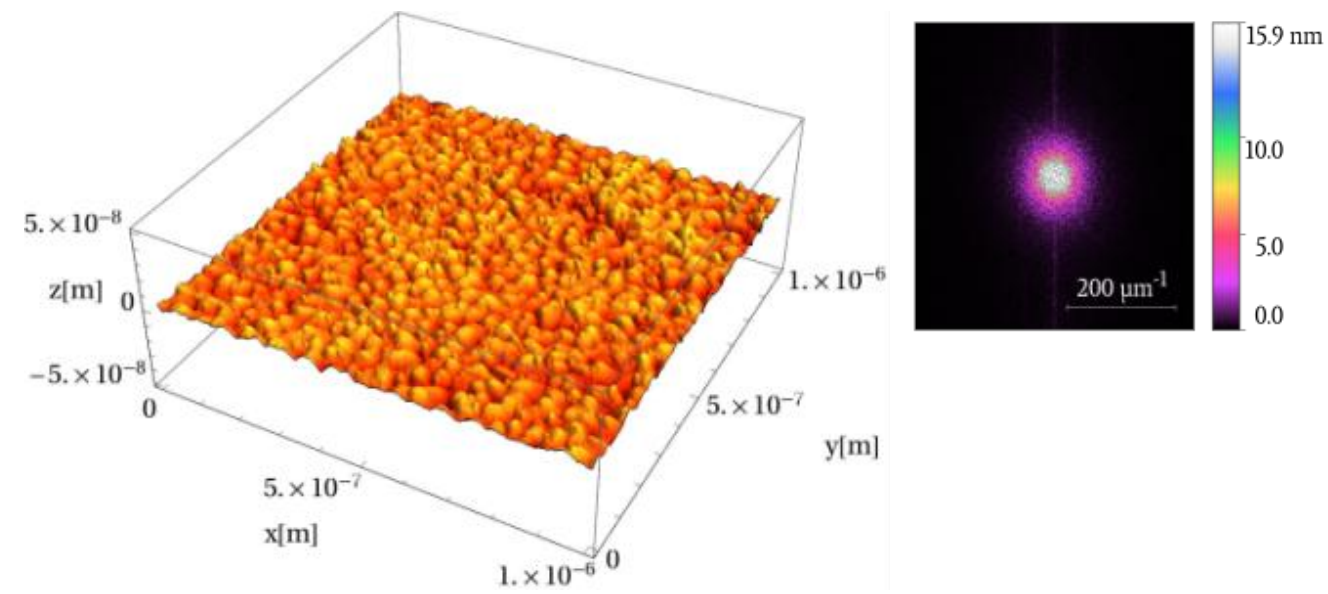

(a)
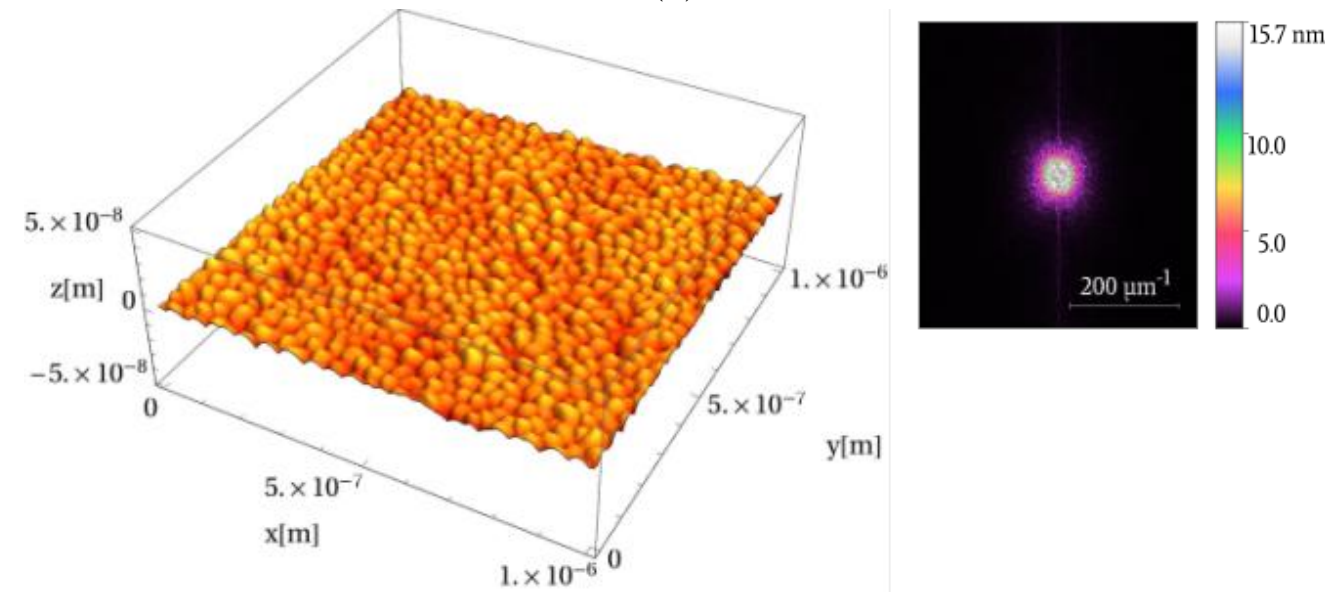

(b)

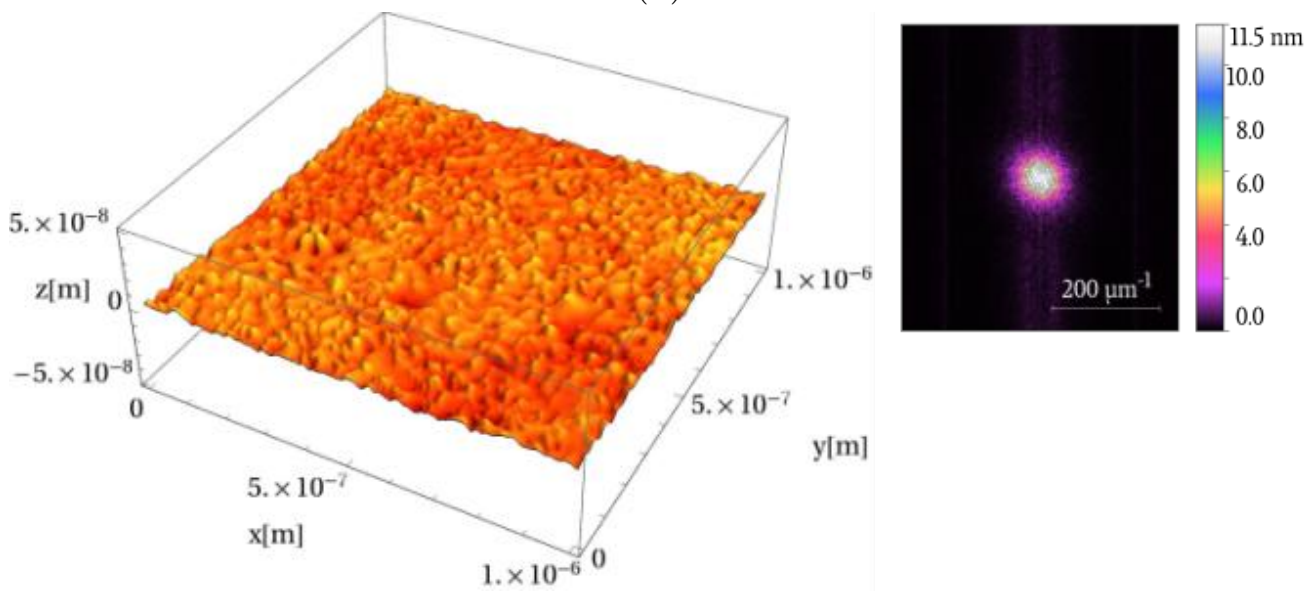

(c)

Figure 6: Left: AFM analyses for the 3 samples; a) Cr/Au prism sample 1, b) Ti/Au prism sample 2 and c) No adhesive layer, pure $\mathrm{Au}$ surface, prism sample 3. Note that the axes are not to scale. Right: The 2D FFT plots for the estimation of the average lateral grain dimensions. 
tions $\left(a_{x}\right.$ and $\left.a_{y}\right)$ are vital in the estimation of $v_{z}$. Though their effects are nominal, they still provide extra information in characterizing these effective layers more accurately and robustly. For this purpose a technique known as the 2D Fast Fourier Transform (2D-FFT) was performed on the AFM data. Figure 6 (right) shows the 2D FFT plots, generated by Gwyddion, for the AFM images of the 3 samples. These plots represent the $z$-axis height correlation intensities in the reciprocal space of the lateral dimensions. The 2D-FFT data provide a unique advantage, as they can be used to obtain the average lateral dimensions of any regular structures when fitted. A Lorentzian fit (Equation 6) seemed to best represent these grains (shown in figure S5). As an additional validation of this approach, similar 2D Lorentzian fits were used in AFM simulators for nanodot oxide growth on silicon substrates , 45 which showed similar semi-ellipsoidal shapes as seen on our Au surfaces.

$$
L(x, y)=\frac{A}{1+\left(a_{x} x\right)^{2}+\left(a_{y} y\right)^{2}} .
$$

For sample 3, instead of grains, wrinkles were seen to be randomly propagating along the surface. These surface topographies seem be the result of this weak magneto-plasmonic adherence causing the unviability of this sample for the use in SPR imaging. Neither of the fits seemed to work with this 2D-FFT data. In addition, sample 3, was also shown to have an uneven surface. This was attributed to the lower surface wettability of NBK-7, forming fewer nucleation sites leading to uneven grain growth before coalescence. This meant that the use of these adhesive metal layers not only helped in Au adherence but also created a more uniform deposition, giving a lower positional variability of its sensitivity on the prism surface.

For sample 1 and 2, the grains are clearly more prominent. An increased number of nucleation sites decreases the energy barrier for the formation of densely packed grains with a narrow grain size distribution, which seemed to differ with respect to the adhesive layer used. $a_{x}$ and $a_{y}$ values for Cr prism sample 1 were estimated to be $30.8 \mathrm{~nm}$ and $29.3 \mathrm{~nm}$ while for Ti prism sample 2 were estimated to be $29.3 \mathrm{~nm}$ and $27.5 \mathrm{~nm}$, respectively, from 
the Lorentzian fits (Equation 6). The values of $a_{x}$ and $a_{y}$ were approximately equal and showed to be at least 3 times larger than $a_{z}$, which satisfied the oblate ellipsoidal condition of the mMGT model $\left(a_{x} \approx a_{y}>a_{z}\right)$.

Finally, the volume fraction $f$ was estimated for samples 1 and 2. For this purpose, initially, the granular volume was calculated of the filtered AFM image (noise and defects removed) using the built-in Gwyddion functions. This was done by first masking the image with a height threshold $\left(a_{z}\right)$, obtained previously. The relative volume fraction was then extracted by dividing the calculated grain volumes from the dimensions of our effective layers $\left(d_{\text {eff }} \times 1 \mu m \times 1 \mu m\right)$. $f$ was calculated to be 0.46 and 0.52 for samples 1 and 2 , respectively. These values were concurrent with what was expected, as sample 2 had slightly more oblate grains within a smaller effective layer. Moreover, even though $f$ has a strong dependence on $a_{x}, a_{y}$ and $a_{z}$, surface coverage of these ellipsoidal grains (essentially directly correlated to the number of nucleation sites) also need to be considered when characterizing this $f$. To further, numerically, understand the surface coverage of these grains an automated Otsu's thresholding method was applied on the AFM images (as seen in figure S6). Even though a slight decrease in surface coverage was seen for sample 1, the relative projected area of the grains was $\approx 51 \%$; almost similar for both sample. Therefore, in our case it can be concluded that the largest contributor to the variation in $f$ is the grain geometry in relation to the effective layer size rather than the grain packing density.

Since all the parameters required for the mMGT were experimentally obtained, the new 5-layer system was used without adjustable parameters to extract the theoretical prism sensitivities. Initially, plasmon curves were obtained at a temperature of $25^{\circ} \mathrm{C}$ as a function of the incident angle (see figure 7). While the model without and effective layer presented deviations from the experimental results for both $\mathrm{Cr}$ and $\mathrm{Ti}$ adhesive layer samples, the mMGT with an effective layer and no adjustable parameters showed strong agreement with the experimental data in the full range accessible for both samples. Moreover, theoretical $\partial R / \partial n$ values of $6570 \% / R I U$ and $6560 \% / R I U$ calculated for $\mathrm{Cr} / \mathrm{Au}$ and $\mathrm{Ti} / \mathrm{Au}$ prism 


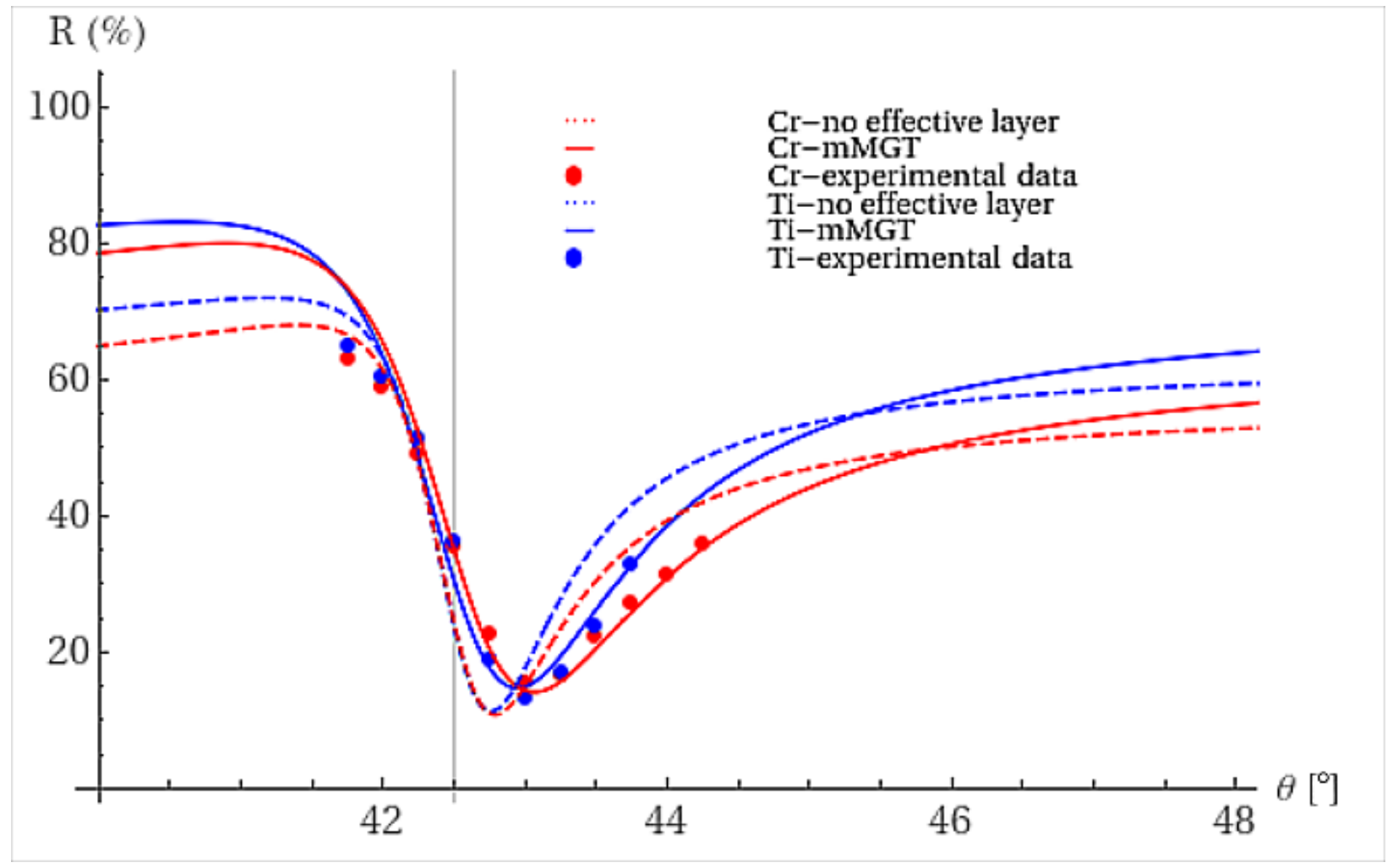

Figure 7: Plasmon curve: Reflectivity (\%) changes with respect to the incident angle $(\theta)$. The experimental points (at $0.25^{\circ}$ increments) are compared with the 2 models with and without effective layer. The model with the inclusion of the surface topography consideration via experimentally derived mMGT shows a stronger agreement with experimental data compared to the model with no effective layer. In both cases, no adjustable parameters were required. The working angle $\left(\theta_{w}\right)$ for both samples was experimentally set to $42.5^{\circ}$ for further analyses. Note that the error bars were within the dot size. 
samples, respectively, matched with the experimentally observable values of $6650 \pm 60 \% / R I U$ and $6490 \pm 120 \% / R I U$ (obtained at $42.5^{\circ}$ as shown in figure 7). The sensitivity losses were captured well with a high degree of accuracy validating our approach for surface topography consideration and their damping effects. In an effort to round off the adhesive layer study, the impact of these surface topographies were included in the re-evaluation of the effect of adhesive layer thicknesses on the prism sensitivities. Figure S7 represents this sensitivities at the achievable working angle $\theta_{w}$ of $42.5^{\circ}$ for both $\mathrm{Cr}$ and $\mathrm{Ti}$ metals. As seen in the Figure S7, with the experimental layer thicknesses $\left(d_{2}=2.4 \mathrm{~nm}\right.$ for $\mathrm{Cr}$ and $d_{2}=3.6 \mathrm{~nm}$ for $\mathrm{Ti}$ ), both theoretical sensitivities are similar reinforcing the validity of the experimental results which showed comparable $\mathrm{Cr} / \mathrm{Au}$ and $\mathrm{Ti} / \mathrm{Au}$ prism performances. The intersection of the two curves falls close to the optimized adhesive layer thickness of $2 \mathrm{~nm}$ suggesting that either $\mathrm{Cr}$ or Ti metals would be convenient. Above this optimal thickness of $2 \mathrm{~nm}$, the adhesive layer with Ti presents improved sensitivity compared to Cr. Table 4 summarizes the experimental values for surface topography characterization, the working angle $\theta_{w}$, the angle $\theta_{\max }$ (theoretically maximizing the sensitivity) and finally the comparison between experimental and theoretically derived prism sensitivities at $25^{\circ} \mathrm{C}$.

Table 4: Experimentally extracted values $f, a_{x}, a_{y}$, and $a_{z}$ from AFM measurements. Working angle $\theta_{w}$ and the angle $\theta_{\max }$ theoretically maximizing the sensitivity. Finally, the theoretical (mMGT) and experimental sensitivities $\partial R / \partial n$ of SPR imaging prisms. Values obtained and modeled at $25^{\circ} \mathrm{C}$.

\begin{tabular}{lllllllll}
\hline $\begin{array}{l}\text { Sample } \\
\text { Units }\end{array}$ & $f$ & $\begin{array}{l}a_{x} \\
{[\mathbf{n m}]}\end{array}$ & $\begin{array}{l}a_{y} \\
{[\mathbf{n m}]}\end{array}$ & $\begin{array}{l}a_{z} \\
{[\mathbf{n m}]}\end{array}$ & $\begin{array}{l}\theta_{w} \\
{\left[{ }^{\circ}\right]}\end{array}$ & $\begin{array}{l}\theta_{\max } \\
{\left[{ }^{\circ}\right]}\end{array}$ & $\begin{array}{l}\text { Theory } \\
{[\% / \mathbf{R I U}]}\end{array}$ & $\begin{array}{l}\text { Experiment } \\
{[\% / R I U]}\end{array}$ \\
\hline \hline $1-\mathrm{Cr} / \mathrm{Au}$ & 0.46 & 30.8 & 29.3 & 5.4 & 42.5 & 42.43 & 6570 & $6650 \pm 60$ \\
\hline $2-\mathrm{Ti} / \mathrm{Au}$ & 0.52 & 29.3 & 27.5 & 4.7 & 42.5 & 42.39 & 6560 & $6490 \pm 120$ \\
\hline
\end{tabular}

Based on this model, the impact of surface topography on prism sensitivity is governed by three parameter; $a_{x, y}, a_{z}$ and $f$, which represent the grain geometry, volume and distribution. To better understand their individual impacts, the maximized $\partial R / \partial n$ (at $\theta_{w}=$ $\left.\theta_{\max }\right)$ were obtained by constraining the other parameters close to their experimental derived counterparts $\left(a_{x, y}, a_{z}\right.$ and $f$ were constrained to $5 \mathrm{~nm}, 30 \mathrm{~nm}$ and 0.5 , respectively). 
$\partial R / \partial n$ were then extracted with respect to these individual parameters. For the parameter $a_{z}$ (figure S8a), the performance showed a significantly higher impact compared to the lateral geometry $a_{x, y}$ parameter (figure $\mathrm{S} 8 \mathrm{~b}$ ). It is clear that the prism sensitivities could be improved significantly with flatter oblate ellipsoids $\left(v_{z} \rightarrow 1\right.$ or $\left.a_{z} / a_{x, y} \rightarrow 0\right)$. In contrast, as the effective layer thickness gets smaller, with increasingly oblate spheroids, $f$ being a relative volume fraction, would increase. This would be counter productive, as an increase in $f$ showed to create a decrease in the sensitivity $\partial R / \partial n$ (figure S8c). Meaning that less densely packed grains (smaller surface coverage) should help in achieving higher sensitivities in this case, as it would also mean less grain boundaries leading to lower damping effects.

Moreover, sample 2 gave high variability in its sensitivity measurements coupled with a slightly higher noise and signal drift (which can be seen in figure S2). This, however, was not attributed to the grains. It is more likely that this variability is due to the relatively weaker adherence between $\mathrm{Ti}$ and $\mathrm{Au}$ compared to $\mathrm{Cr}$. The high tendency for $\mathrm{Cr}$ to form an oxide leads to a stronger $\mathrm{Cr}-\mathrm{O}$ chemical bond. Such bond strengths increase its adhesive properties in contrast to Ti. Furthermore, the higher diffusivity of $\mathrm{Cr}$ compared to Ti leads to $\mathrm{Cr}-\mathrm{Au}$ inter-diffusion. Here, the number of $\mathrm{Cr}-\mathrm{Au}$ bonds could be speculated to exceed the number of Ti-Au bonds, where the chemical bonding could only happen at the surface interface. $\underline{42}$ Accordingly, even though slightly higher sensitivities could be theoretically achieved using Ti/Au prism 2 (at $\theta_{\max }$ ), Cr/Au prism samples prove to be the better choice. As they show lower adhesive layer thickness over-shots during prism preparation and lower sensitivity variabilities, noise and signal drift during utilisation. This make them the better option for opto-eN applications, providing better, reproducible, results with a higher level of stability and prolonged efficacy.

\section{Effect of temperature on prism sensitivity}

To understand the effect of temperature on the prism sensitivity and to further validate the surface topography consideration, the temperature dependence of the model was capitalized 


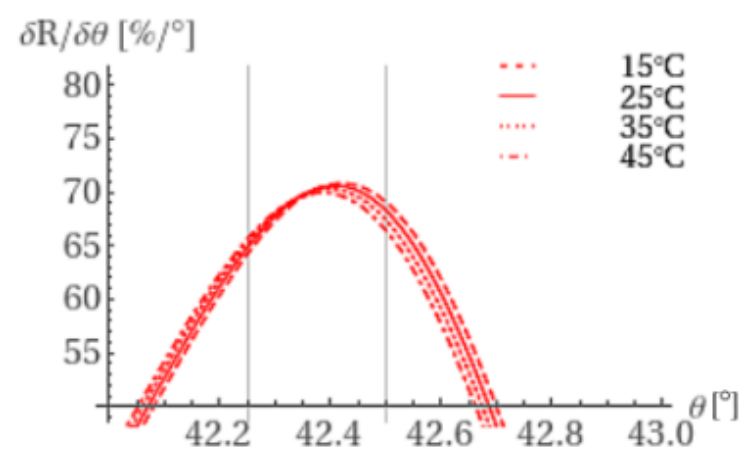

(a) Temperature evolution of $\partial R / \partial \theta$ curves for $\mathrm{Cr}$ adhesive layer prism. Vertical lines: working angles $\theta_{w}$ set before $\left(42.25^{\circ}\right)$ and after $\left(42.5^{\circ}\right) \theta_{\max }$ the angle maximizing the sensitivity.

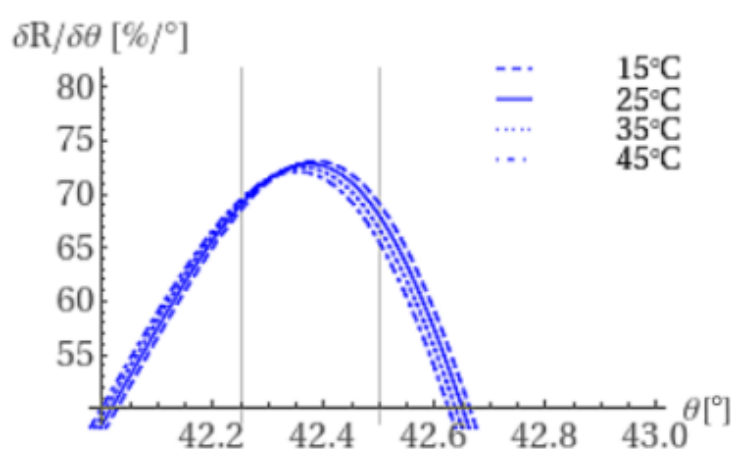

(b) Temperature evolution of $\partial R / \partial \theta$ curves for $\mathrm{Ti}$ adhesive layer prism. Vertical lines: working angles $\theta_{w}$ set before $\left(42.25^{\circ}\right)$ and after $\left(42.5^{\circ}\right) \theta_{\max }$ the angle maximizing the sensitivity.

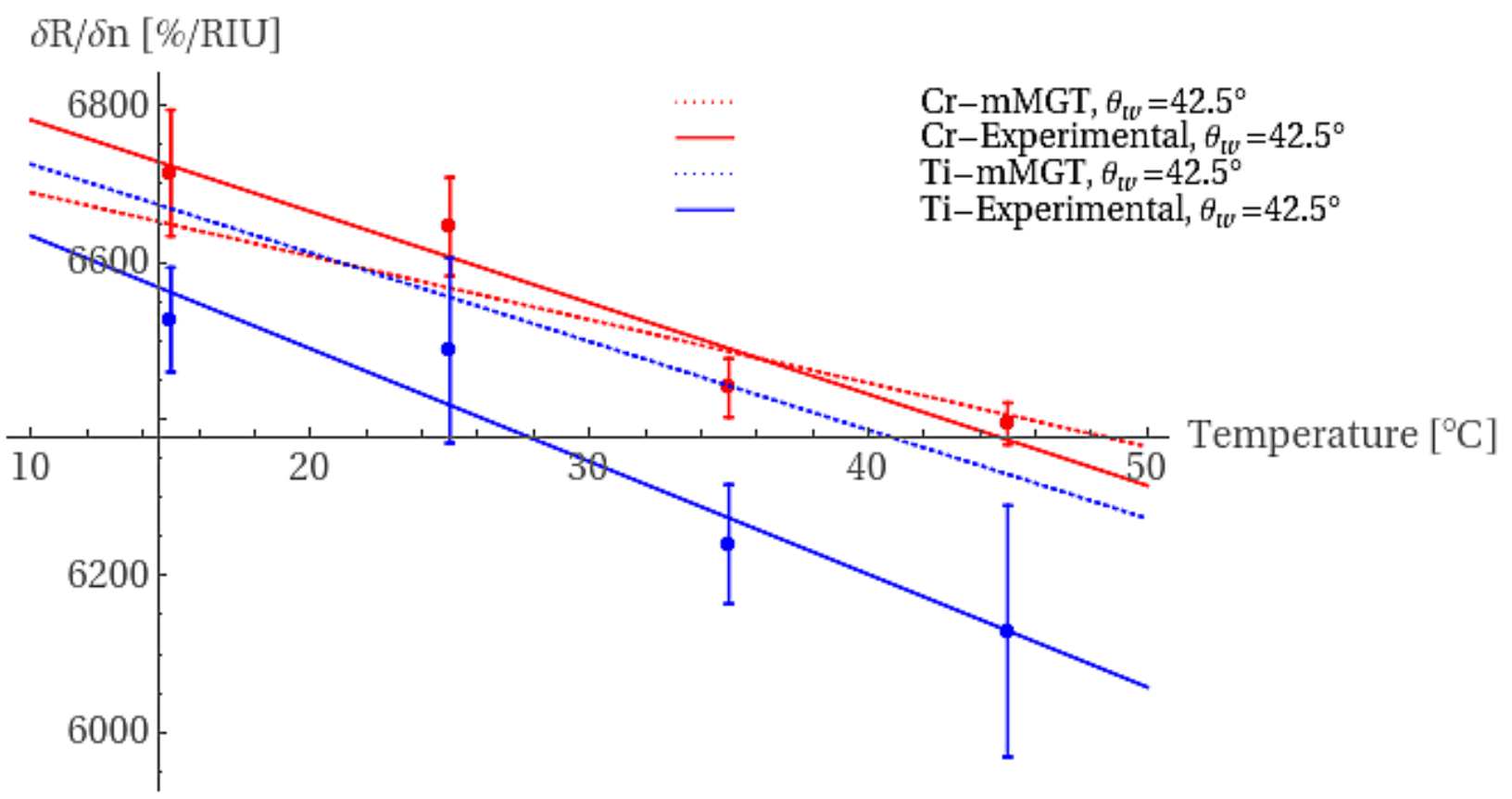

(c) Sensitivity $\partial R / \partial n$ variations with temperature for $\mathrm{Cr}$ and $\mathrm{Ti}$ adhesive layer prism samples. The working angle $\theta_{w}$ was set to $42.5^{\circ}$ experimentally, as the closest achievable angle to $\theta_{\max }$.

Figure 8: The effect of temperature on the sensitivity of the SPR imaging prisms and its relationship to the working angle $\theta_{w}$. 
upon. Four temperature points $\left(15^{\circ} \mathrm{C}, 25^{\circ} \mathrm{C}, 35^{\circ} \mathrm{C}\right.$ and $\left.45^{\circ} \mathrm{C}\right)$ were studied, in accordance with experimental set-up limitations, to mimic in-situ variations in temperature. The working angle $\theta_{w}$ was set at $42.5^{\circ}$ in our case, close to the maximized $\partial R / \partial \theta$. However, as seen in figures $8 \mathrm{a}$ and $8 \mathrm{~b}$ the experimentally achievable $\theta_{w}$ was not equal to $\theta_{\max }$ the angle maximizing the sensitivity. This resulted in a slight increase in the rate of loss of $\partial R / \partial n$ for sample 2 as $\theta_{w}$ was farther away of $\theta_{\max }$ than for sample 1 (see Table 4). The models seemed to correlate well with experimentally obtained values, where sensitivities were calculated at the experimental working angle $\theta_{w}$ (figure 8c). Sample 1 showed a better fit with the theory, showing a maximum variation of $<1 \%$ from the experimental data. On the other hand, sample 2 performed a little worse, which was expected, due to its larger experimental variability. Nevertheless, the maximum theoretical variation was still only at $3 \%$, this too at higher temperatures. This was a significant improvement from the initial $20 \%$ variation from our 4-layer (no effective layer) model. The rate of loss of $\partial R / \partial n$ with respect to an increase in temperature was seen to reduce by moving closer to $\theta_{\max }$ (when $\theta_{w}>\theta_{\max }$ ). For $\theta_{w}<\theta_{\text {max }}$, the relationship seems to reverse, with $\partial R / \partial n$ increasing with temperature (figure S9c]. Temperature showed a more compounded relationship with sensitivity whereas for reflectivity measurements, at a constant $\theta_{w}$, the signal was seen to drift, non-linearly; decreasing at $\approx 0.04 \% /{ }^{\circ} \mathrm{C}$ for an increase in temperature (figure $\mathrm{S} 9 \mathrm{~b}$ ). But even when $\theta_{\max }$ was considered, the overall sensitivity of the system was seen to reduce slightly (figure S9c and S9a), with better sensitivities obtained at lower temperatures. This identified linear relationship could prove useful in temperature correction mechanisms in the opto-eN, however, a better alternative seems to be a new calibration of the working angle $\theta_{w}$ close to $\theta_{\max }$ for in-situ temperature changes larger than $10^{\circ} \mathrm{C}$. 


\section{Conclusion}

In this study, we have first established a novel method in characterizing the optical performance of SPR imaging prisms in gas phase that is independent of the nature of the carrier gases. This is significant, as it would allow for a robust performance measure used for calibration of the prism and the subsequent immobilized sensor array of the opto-eNs; while also enabling the accurate compensation for environmental variation of the carrier gas, which is especially relevant for real world measurements that vary seasonally and regionally. Secondly, using this sensitivity parameter, the effect of two different adhesive layers, namely $\mathrm{Cr}$ and $\mathrm{Ti}$ were studied. For a 4-layer model, at similar adhesive layer thicknesses, Ti based prisms showed higher performances. However, significant sensitivity losses were observed, which were attributed to surface topography damping effects caused by regular grain structures. To characterize these structures, a novel 5-layer model was considered incorporating the surface roughness via the mMGT as a new effective layer. This experimentally validated model, showed the importance of the adhesive layer associated grains on the Au surface. The characterization of the effective layer structure from AFM measurements allows for the 5-layer model predictions to compare quantitatively with the experimental results, free from any fitting parameter. In this regards, Ti based prisms showed a larger loss in sensitivity due to their grain geometry and its relative volume fraction, which were experimentally verified. Even though higher sensitivities could still be achieved with Ti based prisms, a higher variability and drift could lead to reproducibility issues compared to the $\mathrm{Cr}$ counterparts. Thirdly, a temperature based validation was carried out, showing a decrease in overall prism sensitivity with an increase in temperature. Finally, it is important to mention that the model developed in this study could be further expanded upon, to aid in optimizing other multi-layer metal structures and their surface topographies for SPR imaging; to better match theory with experimental realities. 


\section{Acknowledgement}

The authors thank Nanosciences Foundation for the PhD scholarship of J. S. Weerakkody and CEA/DGA for the PhD scholarship of S. Brenet. Part of this work has been funded by the BPI France through the project FUI WISE AAP21 Minalogic project. AFM measurements were done at the Nanocharacterization Plateform (PFNC), which is supported by the Research Technologique de Base (RTB) program of the French Ministry of Research. The authors also thank the support from Labex LANEF (ANR-10-LABX-51-01), Labex ARCANE (ANR-12-LABX-003) and CBH-EUR-GS (ANR-17-EURE-0003).

\section{Supporting Information Available}

Additional figures illustrating SPR imaging sensitivities and AFM measurements and analyses. Transfer matrix method and implementation of the theoretical models.

Click to view the supporting information 


\section{References}

(1) Homola, J. Surface Plasmon Resonance Sensors for Detection of Chemical and Biological Species. Chemical Reviews 2008, 108, 462-493.

(2) Scarano, S.; Mascini, M.; Turner, A. P.; Minunni, M. Surface plasmon resonance imaging for affinity-based biosensors. Biosensors and Bioelectronics 2010, 25, 957-966.

(3) Wong, C. L.; Olivo, M. Surface Plasmon Resonance Imaging Sensors: A Review. Plasmonics 2014, 9, 809-824.

(4) Nylander, C.; Liedberg, B.; Lind, T. Gas detection by means of surface plasmon resonance. Sensors and Actuators 1982, 3, 79-88.

(5) Liedberg, B.; Nylander, C.; Lunström, I. Surface plasmon resonance for gas detection and biosensing. Sensors and Actuators 1983, 4, 299-304.

(6) Miwa, S.; Arakawa, T. Selective gas detection by means of surface plasmon resonance sensors. Thin Solid Films 1996, 281-282, 466-468.

(7) Brenet, S.; John-Herpin, A.; Gallat, F.-X.; Musnier, B.; Buhot, A.; Herrier, C.; Rousselle, T.; Livache, T.; Hou, Y. Highly-Selective Optoelectronic Nose Based on Surface Plasmon Resonance Imaging for Sensing Volatile Organic Compounds. Analytical Chemistry 2018, 90, 9879-9887.

(8) Brenet, S.; John-Herpin, A.; Gallat, F.-X.; Buhot, A.; Livache, T.; Herrier, C.; Rousselle, T.; Hou, Y. Development of a novel multiplexed optoelectronic nose for analysis of volatile organic compounds. 2017 ISOCS/IEEE International Symposium on Olfaction and Electronic Nose (ISOEN). 2017.

(9) Weerakkody, J. S.; Hurot, C.; Brenet, S.; Mathey, R.; Raillon, C.; Livache, T.; Buhot, A.; Hou, Y. Opto-electronic nose - temperature and VOC concentration ef- 
fects on the equilibrium response. 2019 IEEE International Symposium on Olfaction and Electronic Nose (ISOEN). 2019.

(10) Aryballe Technologies, https://aryballe-technologies.com/.

(11) Chang, Y.; Tang, N.; Qu, H.; Liu, J.; Zhang, D.; Zhang, H.; Pang, W.; Duan, X. Detection of Volatile Organic Compounds by Self-assembled Monolayer Coated Sensor Array with Concentration-independent Fingerprints. Scientific Reports 2016, 6 .

(12) Kim, H.-J.; Lee, J.-H. Highly sensitive and selective gas sensors using p-type oxide semiconductors: Overview. Sensors and Actuators B: Chemical 2014, 192, 607-627.

(13) Bai, H.; Shi, G. Gas Sensors Based on Conducting Polymers. Sensors 2007, 7, 267-307.

(14) Mascini, M.; Pizzoni, D.; Perez, G.; Chiarappa, E.; Natale, C. D.; Pittia, P.; Compagnone, D. Tailoring gas sensor arrays via the design of short peptides sequences as binding elements. Biosensors and Bioelectronics 2017, 93, 161-169.

(15) Mascini, M.; Gaggiotti, S.; Pelle, F. D.; Wang, J.; Pingarrón, J. M.; Compagnone, D. Hairpin DNA-AuNPs as molecular binding elements for the detection of volatile organic compounds. Biosensors and Bioelectronics 2019, 123, 124-130.

(16) Kuryoz, P. Y.; Poperenko, L. V.; Kravets, V. G. Correlation between dielectric constants and enhancement of surface plasmon resonances for thin gold films. physica status solidi (a) 2013, 210, 2445-2455.

(17) Kano, H.; Kawata, S. Surface-plasmon sensor for absorption-sensitivity enhancement. Applied Optics 1994, 33, 5166.

(18) Abelès, F. Recherches sur la propagation des ondes électromagnétiques sinusoïdales dans les milieux stratifiés. Annales de Physique 1950, 12, 596-640. 
(19) Hansen, W. N. Electric Fields Produced by the Propagation of Plane Coherent Electromagnetic Radiation in a Stratified Medium. Journal of the Optical Society of America 1968, 58, 380 .

(20) Laplatine, L.; Leroy, L.; Calemczuk, R.; Baganizi, D.; Marche, P. N.; Roupioz, Y.; Livache, T. Spatial resolution in prism-based surface plasmon resonance microscopy. Optics Express 2014, 22, 22771.

(21) Rakić, A. D.; Djurišić, A. B.; Elazar, J. M.; Majewski, M. L. Optical properties of metallic films for vertical-cavity optoelectronic devices. Applied Optics 1998, 37, 5271.

(22) Bach, H., Neuroth, N., Eds. The Properties of Optical Glass; Springer Berlin Heidelberg, 1998.

(23) Chen, Y.-J.; Lee, M.-C.; Wang, C.-M. Dielectric function dependence on temperature for Au and Ag. Japanese Journal of Applied Physics 2014, 53, 08MG02.

(24) Ciddor, P. E. Refractive index of air: new equations for the visible and near infrared. Applied Optics 1996, 35, 1566.

(25) Nesterenko, D. V.; Sekkat, Z. Resolution Estimation of the Au, Ag, Cu, and Al Singleand Double-Layer Surface Plasmon Sensors in the Ultraviolet, Visible, and Infrared Regions. Plasmonics 2013, 8, 1585-1595.

(26) Tervo, P.; Katainen, J.; Sesay, A.; Virtanen, V. The development of SPR device for biomarker detection. Laser Applications in Life Sciences. 2010.

(27) Agarwal, S.; Prajapati, Y.; Singh, V. Influence of metal roughness on SPR sensor performance. Optics Communications 2017, 383, 113-118.

(28) Kanso, M.; Cuenot, S.; Louarn, G. Roughness effect on the SPR measurements for an optical fibre configuration: experimental and numerical approaches. Journal of Optics A: Pure and Applied Optics 2007, 9, 586-592. 
(29) Yang, Z.; Gu, D.; Gao, Y. An improved dispersion law of thin metal film and application to the study of surface plasmon resonance phenomenon. Optics Communications 2014, 329, 180-183.

(30) Markel, V. A. Introduction to the Maxwell Garnett approximation: tutorial. Journal of the Optical Society of America A 2016, 33, 1244.

(31) Jackson, J. D. Classical Electrodynamics Third Edition; Wiley, 1998.

(32) Puiu, M.; Bala, C. SPR and SPR Imaging: Recent Trends in Developing Nanodevices for Detection and Real-Time Monitoring of Biomolecular Events. Sensors 2016, 16, 870.

(33) Martin, P. J. Ion-based methods for optical thin film deposition. Journal of Materials Science 1986, 21, 1-25.

(34) Serrano, A.; de la Fuente, O. R.; García, M. A. Extended and localized surface plasmons in annealed Au films on glass substrates. Journal of Applied Physics 2010, 108, 074303.

(35) Yang, C.; Fan, H.; Xi, Y.; Chen, J.; Li, Z. Effects of depositing temperatures on structure and optical properties of $\mathrm{TiO} 2$ film deposited by ion beam assisted electron beam evaporation. Applied Surface Science 2008, 254, 2685-2689.

(36) Zybin, A.; Boecker, D.; Mirsky, V. M.; Niemax, K. Enhancement of the Detection Power of Surface Plasmon Resonance Measurements by Optimization of the Reflection Angle. Analytical Chemistry 2007, 79, 4233-4236.

(37) Hauf, W.; Grigull, U. Advances in Heat Transfer; Elsevier, 1970; pp 133-366.

(38) Sang, B. H.; Jeon, T.-I. Pressure-dependent refractive indices of gases by THz timedomain spectroscopy. Optics Express 2016, 24, 29040.

(39) Ghorbanpour, M.; Falamaki, C. A novel method for the production of highly adherent $\mathrm{Au}$ layers on glass substrates used in surface plasmon resonance analysis: substitution of 
Cr or Ti intermediate layers with Ag layer followed by an optimal annealing treatment. Journal of Nanostructure in Chemistry 2013, 3, 66.

(40) Sexton, B.; Feltis, B.; Davis, T. Characterisation of gold surface plasmon resonance sensor substrates. Sensors and Actuators A: Physical 2008, 141, 471-475.

(41) Roxworthy, B. J.; Toussaint, K. C. Plasmonic nanotweezers: strong influence of adhesion layer and nanostructure orientation on trapping performance. Optics Express 2012, 20, 9591.

(42) Todeschini, M.; da Silva Fanta, A. B.; Jensen, F.; Wagner, J. B.; Han, A. Influence of Ti and Cr Adhesion Layers on Ultrathin Au Films. ACS Applied Materials $\&$ Interfaces 2017, 9, 37374-37385.

(43) Haynes, W. CRC Handbook of Chemistry and Physics; CRC Handbook of Chemistry and Physics; CRC Press, 2011.

(44) Bhushan, B. Modern Tribology Handbook, Two Volume Set; CRC Press, 2000.

(45) Filipovic, L.; Selberherr, S. Monte Carlo Methods and Applications; DE GRUYTER. 


\section{Graphical TOC Entry}

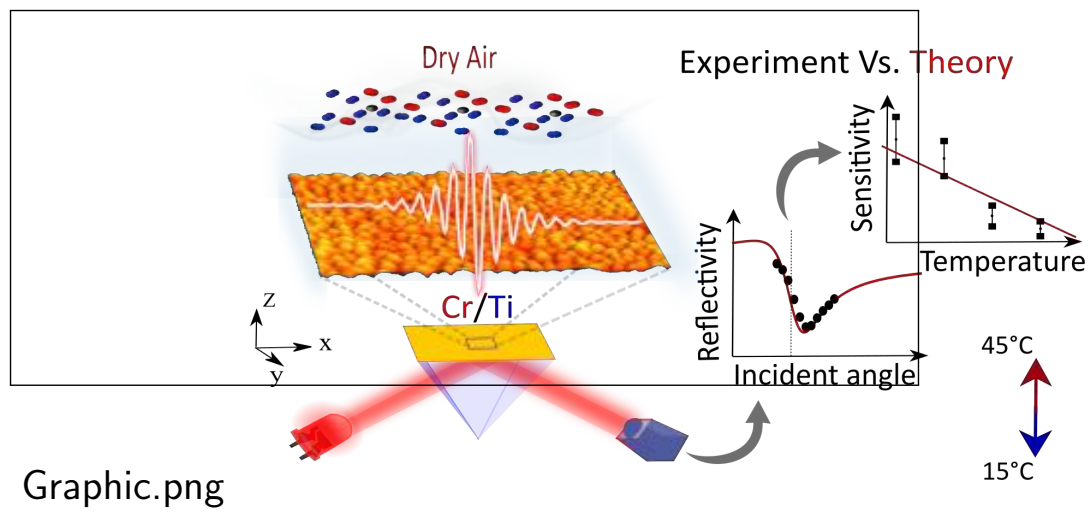

